\title{
COMPACT MODULI SPACES OF DEL PEZZO SURFACES AND KÄHLER-EINSTEIN METRICS
}

\author{
YUJI ODAKA, CRISTIANO SPOTTI, AND SONG SUN
}

\begin{abstract}
We prove that the Gromov-Hausdorff compactification of the moduli space of Kähler-Einstein Del Pezzo surfaces in each degree agrees with certain algebro-geometric compactification. In particular, this recovers Tian's theorem on the existence of Kähler-Einstein metrics on smooth Del Pezzo surfaces and classifies all the degenerations of such metrics. The proof is based on a combination of both algebraic and differential geometric techniques.
\end{abstract}

\section{Contents}

1. Introduction

2. General results on the Gromov-Hausdorff limits

3. Algebro-geometric properties of log Del Pezzo surfaces

3.1. Classification of mildly singular log Del Pezzo surfaces

3.2. CM line bundle comparison

3.3. Semi-universal $\mathbb{Q}$-Gorenstein deformations

3.4. Moduli spaces

4. The cases of degree four and three

4.1. Degree four case

4.2. Degree three case

5. The cases of degree two and one

5.1. More detailed study on Gromov-Hausdorff limits

5.2. Degree two case

5.3. Degree one case

6. Further discussion

6.1. Some remarks

6.2. On compact moduli spaces

References

\section{IntRoduction}

For each positive integer $d$, we denote by $M_{d}^{G H}$ the Gromov-Hausdorff compactification of the moduli space of degree $d$ Kähler-Einstein Del Pezzo surfaces, and denote by $M_{d}^{0}$ the dense subset that parametrizes those smooth surfaces. It is wellknown that for $d \geq 5$ the moduli space is just a single point, so in this paper we will always assume $d \in\{1,2,3,4\}$. By Tian-Yau [75] we know that $M_{d}^{0}$ is at least a non-empty set. By general theory, $M_{d}^{G H}$ is a compact Hausdorff space under the Gromov-Hausdorff topology. By [4, 7, 70, points in $M_{d}^{G H} \backslash M_{d}^{0}$ parametrise certain Kähler-Einstein log Del Pezzo surfaces, and a famous theorem of Tian [70] says that, every smooth Del Pezzo surface admits a Kähler-Einstein metric so that it is actually parametrized in $M_{d}^{0}$. 
In this paper for each $d$ we identify $M_{d}^{G H}$ with certain explicit algebro-geometric moduli space of log Del Pezzo surfaces. The latter is a compact Moishezon analytic space $M_{d}$, which, roughly speaking, parametrizes isomorphism classes of certain $\mathbb{Q}$-Gorenstein smoothable log Del Pezzo surfaces of degree $d$. Notice that there are a-priori several possibilities of such algebro-geometric compactifications of the moduli varieties. On the other hand, the Gromov-Hausdorff compactification is clearly canonical but very non-algebraic and just topological in nature. We refer to 10] as an introductory textbook for those who are not familiar with the GromovHausdorff topology. The following main theorem of the present article builds a bridge between the two notions of moduli spaces.

Theorem 1.1. For each integer $d$, there is a compact moduli algebraic space $1 M_{d}$, that will be constructed explicitly in later sections, and a homeomorphism

$$
\Phi: M_{d}^{G H} \rightarrow M_{d}
$$

such that $[X]$ and $\Phi([X])$ parametrize isomorphic log Del Pezzo surfaces for any $[X] \in M_{d}^{G H}$. Moreover, $M_{d}$ contains a (Zariski) open dense subset which parametrizes all smooth degree d Del Pezzo surfaces.

For the precise formulation, see Section 3.4. Theorem 1.1 immediately implies the above mentioned theorem of Tian, and also classifies all degenerations of KählerEinstein Del Pezzo surfaces which was posed as a problem in [71. When $d=4$ Theorem 1.1 was proved by Mabuchi-Mukai 45, and we shall provide a slightly different proof based on our uniform strategy. For other degrees, there have been partial results by [15, 17, 27, 65, 77] on the existence of Kähler-Einstein metrics on some canonical Del Pezzo surfaces, by calculating $\alpha$-invariant.

A minor point is that the Gromov-Hausdorff topology defined here is slightly different from the standard definition, in that we also remember the complex structure when we talk about convergence. See [23, 66, Chapter 1 and 4] and Section 2 for a related discussion on this. The standard Gromov-Hausdorff compactification is homeomorphic to the quotient of $M_{d}$ by the involution which conjugates the complex structures.

For the proof of Theorem 1.1, we do not need to assume the existence of KählerEinstein metrics on all the smooth Del Pezzo surfaces. The only assumption which we need, and which has been originally proved by Tian-Yau [75, is the following:

Hypothesis 1.2. For each $d \in\{1,2,3,4\}, M_{d}^{0}$ is non-empty as a set.

Given this, the main strategy of proving Theorem 1.1 is as follows:

(1) For each $d$, we construct a natural moduli variety $M_{d}$ with a Zariski open subset $M_{d}^{\text {sm }}$ parametrizing all smooth degree $d$ Del Pezzo surfaces. Moreover, there is a well-defined continuous map $\Phi: M_{d}^{G H} \rightarrow M_{d}$, where we use the Gromov-Hausdorff distance in the domain and the local analytic topology in the target, so that $[X]$ and $\Phi([X])$ parametrize isomorphic log Del Pezzo surfaces for any $[X] \in M_{d}^{G H}$.

(2) $\Phi$ is injective. This follows from the uniqueness theorem of Bando-Mabuchi [8] and its extension to orbifolds.

(3) $\Phi$ is surjective. This follows from the fact that the image of $\Phi$ is open in $M_{d}^{\mathrm{sm}}$ (by the implicit function theorem, see for example [42]) and closed in $M_{d}$ (by the continuity of $\Phi$ in (1)).

(4) Since $M_{d}^{G H}$ is compact and $M_{d}$ is Hausdorff, then $\Phi$ is a homeomorphism.

The main technical part lies in Step (1). For this we need first to investigate Gromov-Hausdorff limits of Kähler-Einstein Del Pezzo surfaces, and then construct

\footnotetext{
${ }^{1}$ For $d \neq 1$, it follows from the construction that $M_{d}$ is actually a projective variety.
} 
a moduli space that includes all the possible limits. The difficulty increases as the degree goes down. When $d=3,4$ we take the classical GIT moduli space on the anti-canonical embedding. For $d=2$ we take the moduli space constructed in 52 (based on Shah's idea 64] which blows up a certain GIT quotient). For $d=1$ we need to combine Shah's method with further modifications suggested by the differential geometric study of Gromov-Hausdorff limits. As far as we are aware, this moduli space is new. We should mention that in the last two cases, $M_{d}$ (and thus $M_{d}^{G H}$ ) contains points that parametrize non-canonical log Del Pezzo surfaces. This disproves a conjecture of Tian in 70, see Remark 5.14. We also remark that Gromov-Hausdorff limits of Kähler-Einstein Del Pezzo surfaces was first studied by Tian in [70, but as we shall see there are some inaccuracies in [70, see Remark 2.8 and Example 5.8 .

Finally we remark that for each $d \in\{1,2,3,4\}$, it is easy to find explicit examples of singular degree $d \mathbb{Q}$-Gorenstein smoothable Kähler-Einstein log Del Pezzo surface by a global quotient construction (see the examples in later sections). Thus one way to avoid assuming Hypothesis 1.2 would be to find a smooth Kähler-Einstein Del Pezzo surface by a gluing construction. For example, it has been proved in 67 that for a Kähler-Einstein log Del Pezzo surface with only nodal singularities and discrete automorphism group, one can glue model Eguchi-Hanson metrics to obtain nearby Kähler-Einstein metrics in the smoothing. This can be applied when $d=3$, since the Cayley cubic (see Section 4) satisfies these assumptions.

The organization of this paper is as follows. In Section 2 we collect the main results that we need on the structure of Gromov-Hausdorff limits, focusing on the two dimensional case. In Section 3 we make an algebro-geometric study of the Gromov-Hausdorff limits, and define precisely the notion of moduli spaces that we use in this paper. Then we reduce the proof of Theorem 1.1 to the construction of moduli spaces in each degree. In later Sections we treat the cases $d \geq 3$ and $d \leq 2$ separately. We also investigate the relation with moduli space of curves, in subsections 5.2.1 5.3.5. In Section 6 we make some further discussions.

\section{Notation:}

A Del Pezzo surface is a smooth projective surface with ample anti-canonical bundle. A log Del Pezzo surface is a normal projective surface with quotient singularities (or equivalently, with log terminal singularities) and ample anti-canonical divisor. For a $\log$ Del Pezzo surface $X$, its degree $\operatorname{deg}(X)$ is the intersection number $K_{X}^{2}$. In general dimensions, a $\mathbb{Q}$-Fano variety means a normal projective variety with log terminal singularities and with $-r K_{X}$ ample for some positive integer $r$. Smallest such $r$ will be called index or Gorenstein index.

Acknowledgements: This work is motivated by the $\mathrm{PhD}$ Thesis of the second named author under the supervision of Professor Simon Donaldson. We would like to thank him for great support. The pre-print version of this paper was written when all the authors were based at Imperial College London. We would also like to thank Professors Jarod Alper, Claudio Arezzo, Paolo Cascini, Ivan Cheltsov, Xiuxiong Chen, Mark Haskins, David Hyeon, Alexander Kasprzyk, Radu Laza, Yongnam Lee, Shigeru Mukai, Hisanori Ohashi, Shingo Taki and Bing Wang for helpful discussions and encouragements. S.S. was partly funded by European Research Council award No 247331. 


\section{General Results on the Gromov-Hausdorff limits}

The main differential geometric ingredient involved in the proof of the main theorem is the study of the structure of Gromov-Hausdorff limits of Kähler-Einstein Del Pezzo surfaces. The following orbifold compactness theorem is well-known.

Proposition 2.1 ([4], 7], 70]). Given a sequence of degree d Kähler-Einstein Del Pezzo surfaces $\left(X_{i}, \omega_{i}, J_{i}\right)$ then, by passing to a subsequence, it converges in the Gromov-Hausdorff sense to a Kähler-Einstein log Del Pezzo surface $\left(X_{\infty}, \omega_{\infty}, J_{\infty}\right)$, and $\operatorname{deg}\left(X_{\infty}\right)=d$.

In 70. Tian found further constraints on the possible singularities that could appear in $X_{\infty}$. We will state a more general theorem and give an alternative proof. First we have (compare also [74]):

Proposition 2.2 (23]). Given a sequence of n-dimensional Kähler-Einstein Fano manifolds $\left(X_{i}, \omega_{i}, J_{i}\right)$, by passing to a subsequence, it converges in the GromovHausdorff sense to a $\mathbb{Q}$-Fano variety $\left(X_{\infty}, J_{\infty}\right)$ endowed with a weak Kähler-Einstein metric $\omega_{\infty}$ (cf. [24]). Moreover, there exist integers $k$ and $N$, depending only on $n$, so that we could embed $X_{i}(i \in \mathbb{N} \cup\{\infty\})$ into $\mathbb{P}^{N}$ using orthonormal basis of $H^{0}\left(X_{i},-k K_{X_{i}}\right)$ with respect to the Hermitian metric defined by $\omega_{i}$, and $X_{i}$ converges to $X_{\infty}$ as varieties in $\mathbb{P}^{N}$.

Here one can think of the convergence as varieties in $\mathbb{P}^{N}$ as the convergence of defining polynomials. Notice that the orbifold property in Proposition 2.1 also follows naturally from Proposition 2.2 since by Kawamata's theorem [36] that a two dimensional log terminal singularity is a quotient singularity.

We will treat singular varieties that come from certain limits of smooth ones. The following algebro-geometric notion is very natural from the point of view of minimal model program, and will be shown to be also naturally satisfied by the above limit $X_{\infty}$.

Definition 2.3. Let $X$ be a $\mathbb{Q}$-Fano variety. We say $X$ is $\mathbb{Q}$-Gorenstein smoothable if there exists a deformation $\pi: \mathcal{X} \rightarrow \Delta \ni 0$ of $X$ over a smooth curve germ $\Delta$ such that $\mathcal{X}_{0}=X$, the general fibre is smooth and $K_{\mathcal{X}}$ is $\mathbb{Q}$-Cartier.

Lemma 2.4. $X_{\infty}$ is $\mathbb{Q}$-Gorenstein smoothable.

Proof. By Proposition 2.2 and general theory we can find a family of varieties $\pi_{2}: \mathcal{X} \subset \mathbb{P}^{N} \times \Delta \rightarrow \Delta$ in $\mathbb{P}^{N}$ where for $t \neq 0 \mathcal{X}_{t}$ is smooth and $\mathcal{X}_{0}$ is the variety $X_{\infty}$. Indeed, for a morphism from $\Delta$ to the Hilbert scheme which sends 0 to $X_{\infty}$ (embedded by $\left|-k K_{X_{\infty}}\right|$ ) and contains $X_{i}$ (embedded by $\left|-k K_{X_{i}}\right|$ as well) for one sufficiently large $i$, we can construct the required family by pulling back the total space and take its normalization if necessary. Denote the other projection map by $\pi_{1}: \mathbb{P}^{N} \times \Delta \rightarrow \mathbb{P}^{N}$, then $-r K_{\mathcal{X}}$ and $\pi_{1}^{*} \mathcal{O}(1)$ agrees up to a pull back from the base. Thus $-r K_{\mathcal{X}}$ is Cartier and so $X_{\infty}$ is $\mathbb{Q}$-Gorenstein smoothable.

Note that the above proof does not use the $\mathbb{Q}$-Gorenstein property of the normal central fiber, although in our case, we knew it by Proposition 2.2 . We only need a relatively ample linear line bundle and the normality assumption of the total space and the central fiber. The definition of $\mathbb{Q}$-Gorenstein smoothability can be obviously defined also for local singularities, and for a $\mathbb{Q}$-Gorenstein smoothable $\mathbb{Q}$-Fano manifold, all its singularities must also be $\mathbb{Q}$-Gorenstein smoothable. On the other hand, it is proved in 30 that a log Del Pezzo surface with $\mathbb{Q}$-Gorenstein smoothable singularities is $\mathbb{Q}$-Gorenstein smoothable. In dimension two, $\mathbb{Q}$-Gorenstein smoothable quotient singularities are also commonly called "T-singularities". The 
classification of $T$-singularities is well-known, see [39, [47 for example. So combining the above discussions we obtain:

Theorem 2.5 ([70]). The Gromov-Hausdorff limit $\left(X_{\infty}, J_{\infty}\right)$ of a sequence of Kähler-Einstein Del Pezzo surfaces is a Kähler-Einstein log Del Pezzo surface with singularities either canonical (i.e. ADE singularities) or cyclic quotients of type $\frac{1}{d n^{2}}(1$, dna -1$)$ with $(a, n)=1(1 \leq a<n)$.

Remark 2.6. For sake of completeness, even if we will not use it in our proof, we should remark that it is known that local smoothings of T-singularities admit asymptotically conical Calabi-Yau metrics [40, 69. It is then natural to expect from a metric perspective the following picture: given a sequence $\left(X_{i}, \omega_{i}\right)$ of degree $d$ Kähler-Einstein Del Pezzo surfaces Gromov-Hausdorff converging to a singular $\left(X_{\infty}, \omega_{\infty}\right)$ and choose $p_{\infty} \in \operatorname{Sing}\left(X_{\infty}\right)$, then there exists a sequence of points $p_{i} \in$ $X_{i} \rightarrow p_{\infty} \in X_{\infty}$ and scaling parameters $\lambda_{i} \rightarrow+\infty$ such that $\left(X_{i}, p_{i}, \lambda_{i} \omega_{i}\right)$ converges in the pointed Gromov-Hausdorff sense to an asymptotically conical Calabi-Yau metric on a smoothing of the T-singularity at $p_{\infty}$.

Next, we can use the Bishop-Gromov volume comparison Theorem to control the order of the orbifold group at each point.

Theorem 2.7 ([70]). Let $(X, \omega)$ be a Kähler-Einstein log Del Pezzo surface and let $\Gamma_{p} \subseteq U(2)$ be the orbifold group at a point $p \in X$. Then

$$
\left|\Gamma_{p}\right| \operatorname{deg}(X)<12,
$$

Proof. Without loss of generality we may normalize the metric so that $\operatorname{Ric}(\omega)=3 \omega$. The Bishop-Gromov volume comparison extends without difficulty to orbifolds 12, so for all $p \in X$ the function $\frac{\operatorname{Vol}(B(p, r))}{\operatorname{Vol}(\bar{B}(r))}$ is decreasing in $r$, where $\bar{B}(r)$ is the ball of radius $r$ in the standard four sphere $S^{4}(1)$. As $r$ tends to zero the function converges to $1 /\left|\Gamma_{p}\right|$, and for sufficiently large $r$ the function is constant $\operatorname{Vol}(X, \omega) / \operatorname{Vol}\left(S^{4}(1)\right)$. So $\operatorname{Vol}(X, \omega)\left|\Gamma_{p}\right| \leq \operatorname{Vol}\left(S^{4}(1)\right)$. The normalization condition $\operatorname{Ric}(g)=3 g$ implies that $[\omega]=\frac{2 \pi}{3} c_{1}(X)$. So $\operatorname{Vol}(X, \omega)=\int_{X} \frac{\omega^{2}}{2}=\frac{2 \pi^{2}}{9} \operatorname{deg}(X)$. Then, using the fact that $\operatorname{Vol}\left(S^{4}(1)\right)=\frac{8}{3} \pi^{2}$, it is easy to see $\left|\Gamma_{p}\right| \operatorname{deg}(X) \leq 12$. If the equality is achieved, then $X$ must have constant curvature. But since $X$ is Kähler, we have $S(\omega)^{2}=$ $24\left|W^{+}\right|^{2}$, so the scalar curvature vanishes. Contradiction.

Remark 2.8. The two theorems above were essentially known to Tian [70. For the inequality [2.1, the constant on the right hand side was 48 in [70.

By Theorem 2.5 and 2.7 and we have the constraints on the possible singularities that could appear on the Gromov-Hausdorff limit $X_{\propto} 2$

- $\operatorname{deg}=4, X_{\infty}$ is canonical, and can have only $A_{1}$ singularities.

- $\operatorname{deg}=3, X_{\infty}$ is canonical, and can have only $A_{1}$ or $A_{2}$ singularities.

- $\operatorname{deg}=2, X_{\infty}$ can have only $A_{1}, A_{2}, A_{3}, A_{4}$, and $\frac{1}{4}(1,1)$ singularities.

- $\operatorname{deg}=1, X_{\infty}$ can have only $\frac{1}{4}(1,1), \frac{1}{8}(1,3)$, and $\frac{1}{9}(1,2)$ singularities besides $A_{i}(i \leq 10)$ and $D_{4}$ singularities.

For the case when $d \geq 3$, the above classification is already sufficient for our purposes, as canonical log Del Pezzo surfaces are classified (see the next section). When $d \leq 2$ we will make a further study in Section 5 . Now we make a side remark about the Gromov-Hausdorff topology used in this paper. In [66] it is proved that if two Kähler-Einstein log Del Pezzo surfaces are isometric, then the complex structures could be the same or conjugate. For this reason the standard Gromov-Hausdorff

\footnotetext{
${ }^{2}$ Recall that the order of the finite Klein group yielding an $A_{k}$ singularity is $k+1$, a $D_{k}$ singularity is $4(k-2)$, an $E_{6}$ singularity is 24 , an $E_{7}$ singularity is 48 , and an $E_{8}$ singularity is 120 .
} 
distance can not distinguish two conjugate complex structures in general. So in our case there is an easy modification, where we say a sequence $\left(X_{i}, J_{i}, \omega_{i}\right)$ converges to $\left(X_{\infty}, J_{\infty}, \omega_{\infty}\right)$ if it converges in the Gromov-Hausdorff topology and in the sense of Anderson-Tian, i.e. smooth convergence of both the metric and complex structure away from the singularities. The spaces $M_{d}$ appearing in Theorem 1.1 admit an involution given by conjugating the complex structure, and we will identify explicitly this involution for each $d$.

\section{Algebro-geometric properties of log Del Pezzo surfaces}

We continue to study the algebro-geometric properties of $X_{\infty}$ appearing in the last section. These constraints help the construction of the desired moduli spaces in later sections.

3.1. Classification of mildly singular log Del Pezzo surfaces. We first recall some general classification results for log Del Pezzo surfaces with mild singularities. The following is classical.

Theorem 3.1 ([31]). A degreed log Del Pezzo surface with canonical singularities is

- a complete intersection of two quadrics in $\mathbb{P}^{4}$, if $d=4$;

- a cubic hypersurface in $\mathbb{P}^{3}$, if $d=3$;

- a degree 4 hypersurface in $\mathbb{P}(1,1,1,2)$ not passing $[0: 0: 0: 1]$, if $d=2$;

- a degree 6 hypersurface in $\mathbb{P}(1,1,2,3)$ not passing $[0: 0: 1: 0]$ and $[0: 0$ : $0: 1]$, if $d=1$.

Although we will not use it, log Del Pezzo surfaces with Gorenstein index two are also classified, by $[3$ and [55]. In case the degree is one or two, we have:

Theorem 3.2 ([37]). A degree 2 log Del Pezzo surface with Gorenstein index at most two is either a degree 4 hypersurface in $\mathbb{P}(1,1,1,2)$, or a degree 8 hypersurface in $\mathbb{P}(1,1,4,4)$. A degree 1 log Del Pezzo surface with Gorenstein index at most two is a degree 6 hypersurface in $\mathbb{P}(1,1,2,3)$.

Notice that by the restrictions on Gromov-Hausdorff limits of Kähler-Einstein Del Pezzo surfaces discussed in the previous section, we know that the Gorenstein index of such limits is less then or equal to 2 for degree $\geq 2$, and at most 6 in the degree 1 case.

3.2. CM line bundle comparison. In this subsection we study GIT stability of Kähler-Einstein log Del Pezzo surfaces. For smooth Kähler-Einstein manifolds, it is known that they are K-polystable (cf. [73, 68, 44]). This has been generalized to the singular setting in [1], and we state the two dimensional case here:

Theorem 3.3 (11]). A log Del Pezzo surface admitting a Kähler-Einstein metric is K-polystable.

Next we state a general theorem relating K-polystability and usual GIT stabilities, using the CM line bundle of Paul-Tian 60. Recall that the CM line bundle is a line bundle defined on base scheme of each flat family of polarized varieties in terms of the Deligne pairing and if the family is $G$-equivariant with an algebraic group $G$, the line bundle naturally inherits the group action. It gives a GIT weight interpretation to the Donaldson-Futaki invariant whose positivity is roughly the K-stability. A point is that the CM line bundle is not even nef in general so that we cannot apply GIT straightforward. We refere to [60, 61] for more details. 
Theorem 3.4. Let $G$ be a reductive algebraic group without nontrivial characters. Let $\pi:(\mathcal{X}, \mathcal{L}) \rightarrow S$ be a $G$-equivariant polarized projective flat family of equidimensional varieties over a projective scheme. Here "polarized" means that $\mathcal{L}$ is a relatively ample line bundle on $\mathcal{X}$, and "equidimensional" means that all the irreducible components have the same dimension. Suppose that

(1) the Picard rank $\rho(S)$ is one;

(2) there is at least one K-polystable $\left(\mathcal{X}_{t}, \mathcal{L}_{t}\right)$ which degenerates in $S$ via a one parameter subgroup $\lambda$ in $G$, i.e. the corresponding test configuration is not of product type.

Then a point $s \in S$ is GIT (poly, semi)stable if $\left(\mathcal{X}_{s}, \mathcal{L}_{s}\right)$ is $K$-(poly, semi)stable.

Proof. Let $\Lambda_{C M}$ be the CM line bundle 60] over $S$ associated to $\pi$. In general, this is a $G$-linearized $\mathbb{Q}$-line bundle. Let $\Lambda_{0}$ be the positive generator of $\operatorname{Pic}(S)$, then there exists integers $r>0$ and $k$, so that $\Lambda_{C M}^{\otimes r} \cong \Lambda_{0}^{\otimes k}$. The isomorphism is $G$ equivariant by the condition that $G$ has no nontrivial character. On the other hand, from the condition (2), we know that the degree of CM line along the closure of the $\lambda$-orbit is positive. This is because by 78 , the degree is the sum of the DonaldsonFutaki invariant on the two degenerations along $\lambda$ and $\lambda^{-1}$. This implies that the integer $k$ is positive. Therefore, $\Lambda_{C M}^{\otimes r}$ is ample.

If $\pi: \mathcal{X} \rightarrow S$ is the universal polarized family over a Hilbert scheme, and $G$ is the associated special linear group $S L$, then it is known 60] that for any $s \in S$ and one parameter subgroup $\lambda: \mathbb{C}^{*} \rightarrow G$, the associated Donaldson-Futaki invariant 22. $\operatorname{DF}\left(\left(\mathcal{X}_{s}, \mathcal{L}_{s}\right) ; \lambda\right)$ is the GIT weight in the usual sense with respect to the $\mathrm{CM}$ line bundle $\Lambda_{C M}^{\otimes r}$, up to a positive multiple. This fact can be extended to our general family $\pi:(\mathcal{X}, \mathcal{L}) \rightarrow S$ in a straightforward way by considering $G$-equivariant morphism into a certain Hilbert scheme defined by $\left(\mathcal{X},\left(\pi^{*} \Lambda_{0}\right)^{\otimes l} \otimes \mathcal{L}^{\otimes m}\right)$ for $l \gg$ $m \gg 0$. If $\mathcal{X}_{s}$ is reduced, from our equidimensionality assumption on all fibers, we can not get almost trivial test configurations from one parameter subgroup of $G$ (in the sense of [43], 59]). This is because the central fiber of an almost trivial test configuration for a reduced equidimensional variety should have an embedded component. Summarizing up, the conclusion follows from the Hilbert-Mumford numerical criterion.

We believe Theorem 3.4 should have more applications in the explicit study of general cscK metrics beyond our study of log Del Pezzo surfaces in this paper. For instance, there are many examples of equivariant family of polarized varieties parametrized by a projective space or Grassmanian through various covering constructions. In these situations one can always apply Theorem 3.4. We remark that in the above proof what we really need is the CM line bundle to be ample. For example, the following has been known to Paul-Tian long time ago:

Corollary 3.5 ([72]). A hypersurface $X \subseteq \mathbb{P}^{N}$ is Chow polystable (resp. Chow semistable) if $\left(X, \mathcal{O}_{X}(1)\right)$ is K-polystable (resp. K-semistable).

Hence, in particular, combined with [58, Theorem 1.2], it follows that semi-logcanonical hypersurfaces with ample canonical classes and log-terminal Calabi-Yau hypersurfaces are GIT stable. This is just one of the easiest examples of applications of Theorem 3.4 .

We also state the following local version of Theorem 3.4, which we also believe to be a fundamental tool for future developments.

Lemma 3.6. Let $S$ be an affine scheme, and $G$ be a reductive algebraic group acting on $S$ fixing $0 \in S$. Let $\pi:(\mathcal{X}, \mathcal{L}) \rightarrow S$ be a $G$-equivariant polarized flat projective deformation of a K-polystable reduced polarized variety $\left(\mathcal{X}_{0}, \mathcal{L}_{0}\right)$ and suppose all fibers $\mathcal{X}_{s}$ are equidimensional varieties. We assume that if $\left(\mathcal{X}_{s_{1}}, \mathcal{L}_{s_{1}}\right)$ is isomorphic 
to $\left(\mathcal{X}_{s_{2}}, \mathcal{L}_{s_{2}}\right)$ then $s_{2} \in G s_{1}$ and that $G$-action on $S$ is faithful. Then there is an affine neighborhood $S^{\prime}$ of 0 so that the CM line bundle is equivariantly trivial over $S^{\prime}$. For such $S^{\prime}$, it holds that a point $s \in S^{\prime}$ is GIT (poly)stable if $\left(\mathcal{X}_{s}, \mathcal{L}_{s}\right)$ is K-(poly) stable.

This follows from similar arguments as in the proof of Theorem 3.4. and we write a detailed proof here for the convenience of readers.

Proof. First, let us prove that the CM line bundle $\lambda_{C M}$ is locally $G$-equivariant trivial around $0 \in S$. Pick an arbitrary but sufficiently ample $G$-equivariant line bundle $\lambda_{B}$ and put $\lambda_{A}:=\lambda_{C M} \otimes \lambda_{B}$. We can assume $\lambda_{B}$ is also ample. By multiplying an appropriate character of $G$ to change the $G$-linearisations on $\lambda_{A}$ and $\lambda_{B}$ if necessary, we can assume the $G$-action at $\left.\lambda_{A}\right|_{0}$ and $\left.\lambda_{B}\right|_{0}$ is trivial. It is possible since from our assumption, $G$-action on $\left.\lambda_{C M}\right|_{0}$ is trivial. Embed $S$ via $\lambda_{A}$ into projective space and take a compactification simply as the Zariski closure $\bar{S}_{A}$. Then applying the Hilbert-Mumford criterion to $\left(\bar{S}_{A}, \lambda_{A}\right)$, we can see that there is some $G$-invariant section $s_{1}$ which does not vanish at 0 . It is indeed the original definition of GIT semi-stability. Note this implies the equivariant triviality of $\lambda_{A}$ at the locus where $s_{1}$ does not vanish. Do the same for $\lambda_{B}$ and we get $s_{2}$, a $G$-invariant section of $B$ non-vanishing around 0 . Then we let $S^{\prime}$ be the locus where both $s_{1}$ and $s_{2}$ do not vanish. This is of course affine again. Over $S^{\prime}, A$ and $B$ are both equivariantly trivial so is the difference which is exactly our CM line bundle.

Suppose $s \in S^{\prime}$ is not polystable in the GIT sense, although $\left(\mathcal{X}_{s}, \mathcal{L}_{s}\right)$ is $\mathrm{K}$ polystable. The non-polystability implies that it degenerates to $s^{\prime} \in\left(S^{\prime} \backslash G s\right)$ via one parameter subgroup $\lambda: \mathbb{C}^{*} \rightarrow G$. This gives a non product test configuration but its Donaldson-Futaki invariant vanishes, due to the equivariant triviality of the CM line bundle over $S^{\prime}$. This contradicts. So we proved the assertion.

One can often apply this to the versal deformation family, as we do in the Section 3.3. We remark that in general if the base $S$ is replaced by an open analytic subset of $S$, we can also work locally analytically provided $S$ is smooth at 0 . Let $K$ be a maximal compact subgroup of $G$, and $A$ be the tangent space of $S$ at 0 . Since $G$ fixes 0 , it induces a linear $G$ action on $A$. We fix a $K$-invariant Hermitian metric on $A$. Then by standard slice theory for compact group actions we can find a $K$-invariant analytic neighborhood $U$ of 0 in $S$, a ball $B_{r}(0)$ in $A$, and a $K$-equivariant biholomorphic map from $U$ to $B_{r}(0)$. So we can identify $U$ with $B_{r}(0)$, in particular, the CM line bundle $\lambda_{C M}$ restricts to $B_{r}(0)$. Our statement then becomes that a point $s \in B_{r}(0)$ is GIT (poly)stable if $\left(\mathcal{X}_{s}, \mathcal{L}_{s}\right)$ is $\mathrm{K}$-(poly)stable. To prove this, by making $r$ small we may choose a non-vanishing holomorphic section $s$ of $\lambda_{C M}$ over $B_{r}(0)$. Now define $\tilde{s}(x)=\int_{K} g^{-1} \cdot s(g \cdot x) d g$, where $d g$ is a bi-invariant Harr measure on $K$. Then $\tilde{s}$ is a $K$-invariant holomorphic section over $B_{r}(0)$, and hence $G$ - invariant (more precisely, it is invariant in the Lie algebra level since $\left.\operatorname{Lie}(G)=\operatorname{Lie}(K)^{\mathbb{C}}\right)$. On the other hand, since $G$ fixes zero, and $\mathcal{X}_{0}$ is K-polystable, $G$ acts trivially on the fiber of $\lambda_{C M}$ over 0 . So $\tilde{s}(0)=s(0) \neq 0$. By making $r$ smaller again we may assume $\tilde{s}$ is also nowhere vanishing over $B_{r}(0)$. Now suppose $x \in B_{r}(0)$ is not polystable in the GIT sense, then there is a unique polystable orbit $G . x^{\prime}$ in the closure of the $G$ orbit of $x$. Moreover by the Kempf-Ness theorem it is easy to see that we may assume $x^{\prime}$ is also in $B_{r}(0)$ (for example, we can choose $x^{\prime}$ to satisfy the moment map equation $\left.\mu\left(x^{\prime}\right)=0\right)$, and there is a one parameter subgroup $\lambda: \mathbb{C}^{*} \rightarrow G$ that degenerates $x$ to $x^{\prime}$. Since $\tilde{s}$ is non-vanishing and Lie $(G)$ invariant, it follows that the Donaldson-Futaki invariant as the weight of the action of the $\mathbb{C}^{*}$ action on the fiber of $\lambda_{C M}$ over $x^{\prime}$ must vanish. By assumption $\mathcal{X}_{x^{\prime}}$ is not isomorphic to $\mathcal{X}_{s}$, this implies $\mathcal{X}_{x}$ is not K-polystable. 
3.3. Semi-universal $\mathbb{Q}$-Gorenstein deformations. In this subsection we provide some general theory on $\mathbb{Q}$-Gorenstein deformations, continuing Section 2 , Most of the general theory we review in the former half of this subsection should be wellknown to experts of deformation theory but we review them for the convenience. First, the following is well-known, see for example the Main Theorem in page 2 of 47:

Lemma 3.7 ([39, [47]). A T-singularity has a smooth semi-universal $\mathbb{Q}$-Gorenstein deformation.

A $T$-singularity is either Du Val (ADE type), which is a hypersurface singularity in $\mathbb{C}^{3}$ and has a smooth semi-universal $\mathbb{Q}$-Gorenstein deformation space, or a cyclic quotient of type $\frac{1}{d n^{2}}(1, d n a-1)$ with $(a, n)=1$. The latter is the quotient of the Du Val singularity $A_{d n-1}$ by the group $\mathbb{Z} / n \mathbb{Z}$. More precisely, an $A_{d n-1}$ singularity embeds as a hypersurface $z_{1} z_{2}=z_{3}^{d n}$ in $\mathbb{C}^{3}$. The generator $\zeta_{n}$ of $\mathbb{Z} / n \mathbb{Z}$ acts on $\mathbb{C}^{3}$ by $\zeta_{n} \cdot\left(z_{1}, z_{2}, z_{3}\right)=\left(\zeta_{n} z_{1}, \zeta_{n}^{-1} z_{2}, \zeta_{n}^{a} z_{3}\right)$, where $\zeta_{n}$ is the $n$-th root of unity. One can explicitly write down a semi-universal $\mathbb{Q}$-Gorenstein deformation as the family of hypersurfaces in $\mathbb{C}^{3}$ given by $z_{1} z_{2}=z_{3}^{d n}+a_{d-1} z_{3}^{(d-1) n}+\cdots+a_{0}$, see [47]. Then its dimension is $d$.

Moreover, it is also known that T-singularities are the only quotient surface singularities which admit $\mathbb{Q}$-Gorenstein smoothings.

Furthermore, for log Del Pezzo surface $X$ which only has T-singularities, since $H^{2}\left(T_{X}\right)=0$ (cf., e.g., [30, Proposition 3.1]), we know that $X$ has global $\mathbb{Q}$ Gorenstein smoothing. Summarising up, we have

Lemma 3.8 (39], Proposition 3.10 and [30, Proposition 2.2). Let $X$ be a log Del Pezzo surface. Then $X$ is $\mathbb{Q}$-Gorenstein smoothable if and only if it has only T-singularities.

We give more precise structure of the deformations as follows, which should be certainly known to experts.

Lemma 3.9. Let $X$ be a $\mathbb{Q}$-Gorenstein smoothable log Del Pezzo surface with singularities $p_{1}, \cdots, p_{n}$. Then for the $\mathbb{Q}$-Gorenstein deformation tangent space Def $(X)$ of $X$, we have

$$
0 \rightarrow \operatorname{Def}(X) \rightarrow \operatorname{Def}(X) \rightarrow \bigoplus_{i=1}^{n} \operatorname{Def}_{i} \rightarrow 0,
$$

where $\operatorname{Def}^{\prime}(X)$ is the subspace of $\operatorname{Def}(X)$ corresponding to equisingular deformations, and Def $f_{i}$ is the $\mathbb{Q}$-Gorenstein deformation tangent space of the local singularity $p_{i}$. Notice Aut $(X)$ naturally acts on Def $(X)$ as well as on $\operatorname{Def}(X)$.

Moreover, if $A u t(X)$ is reductive group, there is an affine algebraic scheme $(\operatorname{Kur}(X), 0)$ with tangent space $\operatorname{Def}(X)$ at 0 , and a semi-universal $\mathbb{Q}$-Gorenstein family $\mathcal{U} \rightarrow(\operatorname{Kur}(X), 0)$ which is $A u t(X)$-equivariant, and the induced action on $\operatorname{Def}(X)$ is the natural one as above. Here Aut $(X)$ denotes the automorphism group of $X$.

We give a sketch of the proof here. In general there is a tangent-obstruction theory for deformation of singular reduced varieties, with tangent space $\operatorname{Ext}^{1}\left(\Omega_{X}, \mathcal{O}_{X}\right)$ and obstruction space $\operatorname{Ext}^{2}\left(\Omega_{X}, \mathcal{O}_{X}\right)$. Since $X$ has only isolated singularities and $H^{2}\left(X, T_{X}\right)=0$ (cf., [30, Proposition 3.1]) in which the local-to-global obstructions lie in general, we have the following natural exact sequnce due to the local-to-global spectral sequence of Ext:

$$
0 \rightarrow H^{1}\left(T_{X}\right) \hookrightarrow \operatorname{Ext}^{1}\left(\Omega_{X}, \mathcal{O}_{X}\right) \rightarrow \oplus_{x \in X} \mathcal{E} x t^{1}\left(\Omega_{X}, \mathcal{O}_{X}\right) \rightarrow 0 .
$$


It is well-known that $H^{1}\left(T_{X}\right)=D e f^{\prime}(X)$ i.e., it is the first order deformation tangent space of equisingular deformations. The local obstruction for deforming singularities lies in the map

$H^{0}\left(\mathcal{E} x t^{1}\left(\Omega_{X}, \mathcal{O}_{X}\right)\right)=\bigoplus_{i=1}^{n} \mathcal{E} x t_{p_{i}}^{1}\left(\Omega_{X}, \mathcal{O}_{X}\right) \rightarrow H^{0}\left(\mathcal{E} x t^{2}\left(\Omega_{X}, \mathcal{O}_{X}\right)\right)=\bigoplus_{i=1}^{n} \mathcal{E} x t_{p_{i}}^{2}\left(\Omega_{X}, \mathcal{O}_{X}\right)$.

Hence, restricting the above exact sequence to the subspace of $\oplus_{x \in X} \mathcal{E} x t^{1}\left(\Omega_{X}, \mathcal{O}_{X}\right)$ which corresponds to $\mathbb{Q}$-Gorenstein deformation (cf., Lemma [3.7, [39, 3.9(i)]) we are done.

Now let us argue the construction $\operatorname{Kur}(X)$. It follows from general algebraic deformation theory (or the Grauert's construction of analytic semi-universal deformation [28) that there exists a formal semi-universal family $\mathcal{X} \rightarrow \operatorname{Spec}(R)$, where $R$ is the completion of an essentially finite type local ring. By using the Grothendieck existence theorem 25 and the Artin algebraicity theorem [5, we obtain a semiuniversal deformation. Moreover, in the semi-universal deformation, it follows from [39. Theorem 3.9(i)] that $\mathbb{Q}$-Gorenstein deformation corresponds to one irreducible component.

As we stated, we can even take semi-universal $\mathbb{Q}$-Gorenstein deformation space $\operatorname{Kur}(X)$ as an $\operatorname{Aut}(X)$-equivariant affine scheme i.e., Aut $(X)$ acts on both the total space of the semi-universal deformation above $\operatorname{Kur}(X)$ and $\operatorname{Kur}(X)$ equivariantly while the projection is equivariant. Indeed, it follows from Luna étale slice theorem which we apply to Hilbert schemes (see [2, especially section 2]) combined with [39, Theorem 3.9(i)].

We remark that for our main applications in this paper we will only need the existence of the versal deformation as an analytic germ, in which case we do not have the action of $\operatorname{Aut}(X)$, but only a holomorphic action of $K$ for a maximal compact subgroup of $\operatorname{Aut}(X)$. This follows from the equivariant version of the construction of Grauert 28] (cf., also 62]).

Now we study a particular example, which we will use in Section 5]

Example 3.10. Let $X_{1}^{T}$ be the quotient of $\mathbb{P}^{2}$ by $\mathbb{Z} / 9 \mathbb{Z}$, where the generator $\xi$ of $\mathbb{Z} / 9 \mathbb{Z}$ acts by $\zeta_{9} \cdot\left[z_{1}: z_{2}: z_{3}\right]=\left[z_{1}: \zeta_{9} z_{2}: \zeta_{9}^{-1} z_{3}\right]$, and $\zeta_{9}$ is the primitive ninth root of unity. Then $X_{1}^{T}$ is a degree one log Del Pezzo surface, with one $A_{8}$ singularity at $[1: 0: 0]$ and two $\frac{1}{9}(1,2)$ singularities at $[0: 1: 0]$ and $[0: 0: 1]$. In particular it is $\mathbb{Q}$-Gorenstein smoothable and has Gorenstein index 3 . Note the Fubini-Study metric on $\mathbb{P}^{2}$ descends to a Kähler-Einstein metric. Since this metric has constant positive bisectional curvature, the cohomology group $H_{\text {orb }}^{1}\left(X, T_{X}\right)$ (the space of harmonic $T_{X}$-valued $(0,1)$ forms on the orbifold) vanishes (see for example, Proposition 9.4 in [19]), so by the obvious orbifold generalization of the KodairaSpencer theory $X_{1}^{T}$ has no equisingular deformations. By the above general theory and a dimension counting using the Main Theorem in [4], we have a decomposition

$$
\operatorname{Def}\left(X_{1}^{T}\right)=D e f_{1} \oplus D e f_{2} \oplus D e f_{3},
$$

where Defi is the $\mathbb{Q}$-Gorenstein deformation tangent space of the local singularity $p_{i}$. It is not hard to see that the connected component of the automorphism group is $A u t^{0}\left(X_{1}^{T}\right)=\left(\mathbb{C}^{*}\right)^{2}$. We want to identify its action on $\operatorname{Def}\left(X_{1}^{T}\right)$. We first choose coordinates on $A u t^{0}\left(X_{1}^{T}\right)$ so that $\lambda=\left(\lambda_{1}, \lambda_{2}\right)$ acts on $X_{1}^{t}=\mathbb{P}^{2} /(\mathbb{Z} / 9 \mathbb{Z})$ by $\lambda .\left[z_{1}\right.$ : $\left.z_{2}: z_{3}\right]=\left[\lambda_{1} z_{1}: \lambda_{2} z_{2}: z_{3}\right]$. Around $p_{3}$ we may choose affine coordinate $y_{1}=$ $z_{1} / z_{3}$, and $y_{2}=z_{2} / z_{3}$. So the action of $\mathbb{Z} / 9 \mathbb{Z}$ is given by $\xi \cdot\left(y_{1}, y_{2}\right)=\left(\zeta_{9} y_{1}, \zeta_{9}^{2} y_{2}\right)$, which is the standard model for the $\frac{1}{9}(1,2)$ singularity. The action of $\left(\mathbb{C}^{*}\right)^{2}$ is then $\lambda .\left(y_{1}, y_{2}\right)=\left(\lambda_{1} y_{1}, \lambda_{2} y_{2}\right)$. Now a local deformation of the affine singularity $\frac{1}{9}(1,2)$ can be seen as follows. We embed $\mathbb{C}^{2} /(\mathbb{Z} / 9 \mathbb{Z})$ into $\mathbb{C}^{3} /(\mathbb{Z} / 3 \mathbb{Z})$ by sending $\left(y_{1}, y_{2}\right)$ to $(u, v, w)=\left(y_{1}^{3}, y_{2}^{3}, y_{1} y_{2}\right)$. A versal deformation is given by $u v-w^{3}=s$. The 
induced action of $\left(\mathbb{C}^{*}\right)^{2}$ is then $\lambda . s=\lambda_{1}^{-3} \lambda_{2}^{-3} s$. This is then the weight of the action on Def $f_{3}$. Similarly one can see the weight on Def $f_{2}$ is given by $\lambda_{1}^{-3} \lambda_{2}^{6}$. To see the weight on Def $f_{1}$, we can embed $X_{1}^{T}$ into $\mathbb{P}(1,2,9,9)$ as a hypersurface $x_{3} x_{4}=x_{2}^{9}$, by sending $\left[z_{1}: z_{2}: z_{3}\right]$ to $\left[x_{1}: x_{2}: x_{3}: x_{4}\right]=\left[z_{1}: z_{2} z_{3}: z_{2}^{9}: z_{3}^{9}\right]$. One can easily write down a space of deformations of $X_{1}^{T}$ as $x_{3} x_{4}=x_{2} \Pi_{i=1}^{8}\left(x_{2}+a_{i} x_{i}\right)$. This deformation only partially smoothes the $A_{8}$ singularity, so that Def $f_{1}$ can be identified with the space of all vectors $\left(a_{1}, \cdots, a_{8}\right)$. It is then easy to see the weight of the action of $\lambda$ on Def $f_{1}$ is $\lambda_{1} \lambda_{2}^{-1}$. So we have arrived at:

Lemma 3.11. The action of $A u t^{0}\left(X_{1}^{T}\right)$ on $\operatorname{Def}\left(X_{1}^{T}\right)$ is given by

$$
\lambda .\left(v_{1}, v_{2}, v_{3}\right)=\left(\lambda_{1} \lambda_{2}^{-1} v_{1}, \lambda_{1}^{-3} \lambda_{2}^{6} v_{2}, \lambda_{1}^{-3} \lambda_{2}^{-3} v_{3}\right) .
$$

From Lemma 3.9 we have a linear action of a group $\operatorname{Aut}(X)$ on $\operatorname{Def}(X)$. If $\operatorname{Aut}(X)$ is reductive (for example, when $X$ admits a Kähler-Einstein metric, by Matsushima's theorem [50]), one can take a GIT quotient $\operatorname{Def}(X) / / \operatorname{Aut}(X)$. So we are in the situation of the remark after Lemma 3.6, and locally analytically this can be viewed as a "local" coarse moduli space of $\mathbb{Q}$-Gorenstein deformations of $X$. The following lemma provides a more precise link between the Gromov-Hausdorff convergence and algebraic geometry.

Lemma 3.12. Let $X_{\infty}$ be the Gromov-Hausdorff limit of a sequence of KählerEinstein Del Pezzo surfaces $X_{i}$, then for $i$ sufficiently large we may represent $X_{i}$ by a point $u_{i}$ in an open neighborhood of the GIT quotient $\operatorname{Kur}\left(X_{\infty}\right) / / \operatorname{Aut}\left(X_{\infty}\right)$ (analytically interpreted above as $\operatorname{Def}(X) / / \operatorname{Aut}(X))$ so that $u_{i} \rightarrow 0$ as $i$ goes to infinity.

Proof. From Section 2, we know there are integers $m, N$, such that by passing to a subsequence the surface $X_{i}$ converges to $X_{\infty}$, under the projective embedding into $\mathbb{P}^{N}$ defined by orthonormal section of $H^{0}\left(X_{i},-m K_{X_{i}}\right)$. Since $X_{\infty}$ has reductive automorphism group, we can choose a Luna slice $S$ in the component of the Hilbert scheme corresponding to $\mathbb{Q}$-Gorenstein smoothable deformations of $X_{\infty}$. Hence for $i$ large enough, $X_{i}$ is isomorphic to a surface parametrized by $s_{i}(\in S) \rightarrow 0$. By the versality, shrinking $S$, we have a map $F: S \rightarrow \operatorname{Kur}\left(X_{\infty}\right)$ so that $s$ and $F(s)$ represent isomorphic surfaces. Let $v_{i}=F\left(s_{i}\right)$. Then $v_{i} \rightarrow 0$. Moreover, by the remark after Lemma 3.6, the corresponding point to $v_{i}$ in $\operatorname{Def}(X)$ is polystable for $i$ large, thus its image $u_{i} \in \operatorname{Kur}\left(X_{\infty}\right) / / \operatorname{Aut}\left(X_{\infty}\right)$ represents the same surface $X_{i}$. The conclusion then follows.

3.4. Moduli spaces. In this section we will define precisely what "moduli of Kähler-Einstein $\mathbb{Q}$-Fano varieties" means to us in this paper.

Definition 3.13 (KE moduli stack). We call a moduli algebraic (Artin) stack $\mathcal{M}$ of $\mathbb{Q}$-Gorenstein family of $\mathbb{Q}$-Fano varieties a $K E$ moduli stack if

(1) It has a categorical moduli $M$ in the category of algebraic spaces;

(2) There is an étale covering of $\mathcal{M}$ of the form $\left\{\left[U_{i} / G_{i}\right]\right\}$ with affine algebraic schemes $U_{i}$ and reductive groups $G_{i}$, where there is a $G_{i}$-equivariant $\mathbb{Q}$ Gorenstein flat family of $\mathbb{Q}$-Fano varieties.

(3) Closed orbits of $G_{i} \curvearrowright U_{i}$ correspond to geometric points of $M$, and parametrize $\mathbb{Q}$-Gorenstein smoothable Kähler-Einstein $\mathbb{Q}$-Fano varieties.

We call the categorical moduli in the category of algebraic space $M$ a KE moduli space. If it is an algebraic variety, we also call it KE moduli variety.

For an introduction to the theory of algebraic stacks, one may refer to [9. For the general conjecture and for more details on the existence of KE moduli stack, compare Section 6. For our main purposes in proving Theorem 1.1] we only need a much weaker notion. 
Definition 3.14 (Analytic moduli space). An analytic moduli space of degree $d$ log Del Pezzo surfaces is a compact analytic space $M_{d}$ with the following structures:

(1) We assign to each point in $M_{d}$ a unique isomorphism class of $\mathbb{Q}$-Gorenstein smoothable degree $d$ log Del Pezzo surfaces. For simplicity of notation, we will denote by $[X] \in M_{d}$ a point which corresponds to the isomorphism class of the log Del Pezzo surface $X$.

(2) For each $[X] \in M_{d}$ with $\operatorname{Aut}(X)$ reductive, there is an analytic neighborhood $U$, and a quasi-finite locally bi-holomorphic map $\Phi_{U}$ from $U$ onto an analytic neighborhood of $0 \in \operatorname{Def}(X) / / \operatorname{Aut}(X)$ (where as in the remark after Lemma 3.6, we have chosen a $K$-equivariant identification between analytic neighborhoods in $\operatorname{Kur}(X)$ and $\operatorname{Def}(X))$ such that $\Phi_{U}^{-1}(0)=[X]$ and for any $u \in U$, the surfaces parametrized by $u$ and $\Phi_{U}(u)$ are isomorphic.

Definition 3.15. We say that an analytic moduli space has property (KE) if every surface parametrized by $M_{d}^{G H}$ is isomorphic to one parametrized by some point in $M_{d}$.

Theorem 3.16. For any analytic moduli space $M_{d}$ which has property (KE), there is a homeomorphism from $M_{d}^{G H}$ to $M_{d}$, under the obvious map.

Proof. To carry out the strategy described in the introduction, we just need the natural map from $M_{d}^{G H}$ to $M_{d}$ to be continuous. It suffices to show that if we have a sequence $\left[X_{i}\right] \in M_{d}^{0}$ converges to a point $\left[X_{\infty}\right] \in M_{d}^{G H}$, then $\Phi\left(\left[X_{i}\right]\right)$ converges to $\Phi\left(\left[X_{\infty}\right]\right)$. Unwrapping the definitions, this is exactly Lemma 3.12 .

In later sections 4 and 5 , we will construct the analytic moduli space $M_{d}$ for $\mathbb{Q}$-Gorenstein smoothable cases one-by-one. We will show that these $M_{d}$ 's satisfy property (KE). Moreover they are actually categorical moduli of moduli stacks $\mathcal{M}_{d}$, with a Zariski open subset parametrizing all smooth degree $d$ Del Pezzo surfaces. Thus Theorem 1.1 follows.

\section{The CASEs of Degree FOUR AND THREE}

4.1. Degree four case. In this case Theorem 1.1 has already been proved in [45]. Following the general strategy outlined in the introduction, we give a partially new proof here. Recall that smooth degree 4 Del Pezzo surfaces are realized by the anti-canonical embedding as intersections of two quadrics in $\mathbb{P}^{4}$. So in order to construct a moduli space, it is natural to consider the following GIT picture

$$
\operatorname{PGL}(5 ; \mathbb{C}) \curvearrowright H_{4}=\operatorname{Gr}\left(2, \operatorname{Sym}^{2}\left(\mathbb{C}^{5}\right)\right) \hookrightarrow \mathbb{P}_{*}\left(\Lambda^{2} \operatorname{Sym}^{2}\left(\mathbb{C}^{5}\right)\right)^{3},
$$

with a linearization induced by the Plücker embedding. Here, Gr stands for the Grassmanian.

Theorem 4.1 (Mabuchi-Mukai [45]). An intersection $X$ of two quadrics in $\mathbb{P}^{4}$ is

- stable $\Longleftrightarrow X$ is smooth;

- semistable $\Longleftrightarrow X$ has at worst $A_{1}$ singularities (nodes);

- polystable $\Longleftrightarrow$ the two quadrics are simultaneously diagonalizable, i.e. X is isomorphic to the intersection of quadrics

$$
\left\{\begin{array}{l}
x_{0}^{2}+x_{1}^{2}+x_{2}^{2}+x_{3}^{2}+x_{4}^{2}=0 \\
\lambda_{0} x_{0}^{2}+\lambda_{1} x_{1}^{2}+\lambda_{2} x_{2}^{2}+\lambda_{3} x_{3}^{2}+\lambda_{4} x_{4}^{2}=0
\end{array}\right.
$$

and no three of the $\lambda_{i} s$ are equal (or equivalently, $X$ is either smooth or has exactly two or four $A_{1}$ singularities).

\footnotetext{
${ }^{3}$ In this paper $\mathbb{P}_{*}(V)=\mathbb{P}(V)$ is the covariant projectivization and $\mathbb{P}^{*}(V)=\mathbb{P}\left(V^{*}\right)$ is the contravariant projectivization.
} 
Since two degree four Del Pezzo surfaces as above considered are abstractly biholomorphic if and only if their equations in the above embeddings are transformed by the natural action of an element of $P G L(5 ; \mathbb{C}$ ) (this follows by the very ampleness of the anticanonical line bundle $=$, the GIT quotient

$$
M_{4}:=H_{4}^{s s} / / P G L(5 ; \mathbb{C})
$$

parametrizes (abstract) isomorphism classes of polystable intersections of two quadrics. We remark here that the same reasoning should be applied later in the other degree cases.

Moreover, since $M_{4}$ is naturally coarsely isomorphic to the moduli space of binary quintics on $\mathbb{P}^{1}$, choosing invariants as in 21, Chapter 10.2 , we see that $M_{4}$ is isomorphic to $\mathbb{P}(1,2,3)$ and that the smooth surfaces are parametrized by the Zariski open subset $M_{4}^{\mathrm{sm}} \cong \mathbb{P}(1,2,3) \backslash D$, where $D$ is an ample divisor cut out by the equation $z_{1}^{2}=128 z_{2}$.

The $d=4$ case of Theorem 1.1 then follows from the following:

Theorem 4.2. The above constructed $M_{4}$ is an analytic moduli space with property $(K E)$.

Proof. First we verify that our degenerations of del Pezzo surfaces in $\mathbb{P}^{4}$ can not give any "pathological test configuration" in the sense of [43] (called "almost trivial" test configuration in [59]) whose normalization is trivial. It is due to the following reason. The central fibers of such pathological test configurations is not equidimensional, so it is especially not Cohen-Macaulay. However, the degenerations in $\mathbb{P}^{4}$ that we considered here are all Cohen-Macaulay. This is because, in general, weighted projective spaces only have quotient singularities, and so are Cohen-Macaulay (cf., e.g., 34]). Then the finite times cut by Cartier divisors (hypersurfaces) are inductively Cohen-Macaulay (cf., e.g., [49, page 105]). Later we will use the similar reasoning for other degrees as well.

To check $M_{4}$ is an analytic moduli space, observe that item (1) is obvious, and item (2) follows from the construction of $M_{4}$ as a GIT quotient (the versal family is the universal one over $H_{4}$ ). To see $M_{4}$ has property (KE), we first use Theorem 3.1 to see that any $[X] \in M_{4}^{G H}$ is parametrized by $H_{4}$. Then we apply Theorem 3.3 and Theorem 3.4 (since Picard rank of $H_{4}$ is one, and it is easy to verify the assumptions are satisfied in this case) to see that $[X]$ is parametrized by $M_{4}$.

Clearly $\mathcal{M}_{4}:=\left[H_{4}^{s s} / P G L(5 ; \mathbb{C})\right]$ is a quotient stack, so we conclude that it is indeed a KE moduli stack. We make a few remarks here. First of all, the above arguments actually prove that all degree four Kähler-Einstein log Del Pezzo surfaces are parametrized by $M_{4}$. By Theorem 4.1 the Gromov-Hausdorff limits of smooth Del Pezzo quartics have only an even number of $A_{1}$ singularities. The maximum number of such singularities is four. There is exactly one such surface $X_{4}^{T}$, which is defined by the equations $x_{0} x_{1}=x_{2}^{2}=x_{3} x_{4}$. It is isomorphic to the quotient $\mathbb{P}^{1} \times \mathbb{P}^{1} /(\mathbb{Z} / 2 \mathbb{Z})$, where the generator $\xi$ of $\mathbb{Z} / 2 \mathbb{Z}$ acts as $\xi \cdot\left(z_{1}, z_{2}\right)=\left(-z_{1},-z_{2}\right)$. So it admits an obvious Kähler-Einstein metric.

It is also easy to see that the action of complex conjugation, which sends a Del Pezzo quartic to its complex conjugate, coincides with the natural complex conjugation on $\mathbb{P}(1,2,3)$.

4.2. Degree three case. Recall that smooth degree 3 Del Pezzo surfaces are cubic hypersurfaces in $\mathbb{P}^{3}$. Note that the anti-canonical bundle is very ample. We recall the following classical GIT picture. The group $P G L(4 ; \mathbb{C})$ acts naturally on the space $H_{3}=\mathbb{P}_{*}\left(\operatorname{Sym}^{3}\left(\mathbb{C}^{4}\right)\right) \cong \mathbb{P}^{19}$ of cubic polynomials. 
Theorem 4.3 (Hilbert). A cubic surface $X$ in $\mathbb{P}^{3}$ is

- stable $\Longleftrightarrow X$ has at most singularities of type $A_{1}$;

- semistable $\Longleftrightarrow X$ has at worst singularities of type $A_{1}$ or $A_{2}$;

- strictly polystable $\Longleftrightarrow X$ is isomorphic to the cubic $X_{3}^{T}$ defined by equation $x_{1} x_{2} x_{3}=x_{0}^{3}$. It is not hard to see that $X_{3}^{T}$ has exactly three $A_{2}$ singularities, and is isomorphic to the quotient $\mathbb{P}^{2} /(\mathbb{Z} / 3 \mathbb{Z})$, where the generator $\xi$ of $(\mathbb{Z} / 3 \mathbb{Z})$ acts by $\xi \cdot\left[z_{1}: z_{2}: z_{3}\right]=\left[z_{1}: e^{2 \pi i / 3} z_{2}: e^{-2 \pi i / 3} z_{3}\right]$.

Define the quotient stack $\mathcal{M}_{3}:=\left[H_{3}^{s s} / P G L(4 ; \mathbb{C})\right]$ and the corresponding GIT quotient (or in other word, categorical moduli)

$$
M_{3}:=H_{3}^{s s} / / P G L(4 ; \mathbb{C})
$$

which parametrizes isomorphism classes of polystable cubics. The above Theorem is classical. It was proved by D. Hilbert in his Doctoral dissertation [32. For a modern proof consult 53 . Moreover, by looking at the ring of invariants [63, it is known that

$$
M_{3} \cong \mathbb{P}(1,2,3,4,5),
$$

and that $M_{3}^{\mathrm{sm}} \cong \mathbb{P}(1,2,3,4,5) \backslash D$ where $D$ is the ample divisor of equation $\left(z_{1}^{2}-\right.$ $\left.64 z_{2}\right)^{2}-2^{11}\left(8 z_{4}+z_{1} z_{3}\right)=0$. So $M_{3}^{\mathrm{sm}}$ is Zariski open and parametrizes all smooth cubic surfaces.

Note that we can apply Theorem 3.4 for universal family over $H_{3}$. Thus it follows that $\mathcal{M}_{3}$ is a KE moduli stack and $M_{3}$ is a KE moduli variety.

Observe that a Gromov-Hausdorff limit of smooth Kähler-Einstein cubic surfaces has either exactly three $A_{2}$ singularities or at most four $A_{1}$ singularities. In the former case, it is isomorphic to $X_{3}^{T}$. In the latter case, this is the Cayley's cubic $X_{3}^{C}$ defined by $x_{0} x_{1} x_{2}+x_{1} x_{2} x_{3}+x_{2} x_{3} x_{0}+x_{3} x_{0} x_{1}=0$. It is not hard to see it is isomorphic to the quotient of $X_{6} /(\mathbb{Z} / 2 \mathbb{Z})$, where $X_{6}$ is the degree six Del Pezzo surface, and the action of $\mathbb{Z} / 2 \mathbb{Z}$ is induced by the standard Cremona transformation on $\mathbb{P}^{2}$, i.e., $\left[z_{1}: z_{2}: z_{3}\right] \mapsto\left[z_{1}^{-1}: z_{2}^{-1}: z_{3}^{-1}\right]$. The existence of Kähler-Einstein metrics on $X_{3}^{T}$ and $X_{3}^{C}$ can also be easily seen using the above quotient description.

We remark that it was proved in [20] that a Kähler-Einstein cubic surface must be GIT semistable, and our application of Theorem 3.4 sharpens this. The existence of Kähler-Einstein metrics on cubic surfaces with exactly one $A_{1}$ singularity was proved by [77, using Kähler-Ricci flow on orbifolds and certain calculation of $\alpha$ invariants. In 67, by glueing method we know the existence of Kähler-Einstein metrics on a partial smoothing of the Cayley cubic $X_{3}^{C}$. For general cubics with two or three $A_{1}$ singularities this was previously unknown. Here we actually know that all degree three $\mathbb{Q}$-Gorenstein smoothable Kähler-Einstein log Del Pezzo surfaces are parametrized by $M_{3}$.

As in the degree four case, the action of complex conjugation on $M_{3}$ is also given by the natural anti-holomorphic involution.

\section{The CASES OF DEGREE TWO AND ONE}

5.1. More detailed study on Gromov-Hausdorff limits. When the degree is one or two, there are new difficulties as non-canonical singularities could appear in the Gromov-Hausdorff limits. So the classification of canonical Del Pezzo surfaces (Theorem 3.1) is not enough for our purpose. In degree two by Theorem 2.7 we only need to deal with index 2 log del Pezzo surfaces, which have been classified in [3], 55], 37]. We could simply use these classification results directly, but since our assumption is much more restricted, we provide a more elementary approach which treats both $d=1$ and $d=2$ cases. 
A common feature of the two cases is the existence of a holomorphic involution. For a degree two Del Pezzo surface $X$, it is well-known that the anti-canonical map defines a double cover of $X$ to $\mathbb{P}^{2}$. Therefore $X$ admits an involution $\sigma$ ("Geiser involution") which is simply the deck transformation of the covering map. The fixed locus of $\sigma$ is smooth quartic curve. If $X$ admits a Kähler-Einstein metric $\omega$, then by $8 \omega$ must be invariant under any such $\sigma$. Similarly for a degree one Del Pezzo surface $X$, the linear system $\left|-2 K_{X}\right|$ defines a double cover of $X$ to $\mathbb{P}(1,1,2) \subset \mathbb{P}^{3}$. So $X$ also admits an involution $\sigma$ ("Bertini involution"). Again any such $\sigma$ must preserve the Kähler-Einstein metric if $X$ admits one. The fixed locus of $\sigma$ consists of the point $[0: 0: 1]$ and a sextic in $\mathbb{P}(1,1,2)$.

Lemma 5.1. Suppose a sequence of degree one (or two) Kähler-Einstein Del Pezzo surfaces $\left(X_{i}, \omega_{i}, J_{i}\right)$ converges to a Gromov-Hausdorff limit $\left(X_{\infty}, \omega_{\infty}, J_{\infty}\right)$, then by passing to a subsequence one can take a limit $\sigma_{\infty}$, which is a holomorphic involution on $X_{\infty}$.

Proof. This is certainly well-known. We include a proof here for the convenience of readers. Let $p_{1}, \cdots, p_{n}$ be the singular points of $X_{\infty}$. We denote $\Omega_{r}=X_{\infty} \backslash$ $\cup_{j=1}^{n} B\left(p_{j}, r\right)$. For any $r>0$ small, from the convergence theorem 2.1 we know that for $i$ sufficiently large, there are $\sigma_{i}$ invariant open subsets $\Omega_{i} \subset X_{i}$ and embeddings $f_{i}: \Omega_{i} \rightarrow X_{\infty} \backslash\left\{p_{1}, \cdots, p_{n}\right\}$ such that $\Omega_{r}$ is contained in the image of each $f_{i}$ and $\left(f_{i}^{-1}\right)^{*}\left(\omega_{i}, J_{i}\right)$ converges to $\left(\omega_{\infty}, J_{\infty}\right)$ smoothly. Then, by passing to a subsequence, the isometries $\left(f_{i}^{-1}\right)^{*} \sigma_{i}$ converge to a limit $\sigma_{r, \infty}: \Omega_{r} \rightarrow X_{\infty}$ with $\sigma_{r, \infty}^{*}\left(\omega_{\infty}, J_{\infty}\right)=$ $\left(\omega_{\infty}, J_{\infty}\right)$. Then we can let $r$ tend to zero and choose a diagonal subsequence so that $\sigma_{r, \infty}$ converges to a holomorphic isometry $\sigma_{\infty}$ on $X_{\infty} \backslash\left\{p_{1}, \cdots, p_{n}\right\}$. Then by the Hartog's extension theorem, $\sigma_{\infty}$ extends to a holomorphic isometry on the whole $X_{\infty}$. It is also clear $\sigma_{\infty}^{2}$ is the identity.

Theorem 5.2. In the degree two case, $X_{\infty}$ is either a double cover of $\mathbb{P}^{2}$ branched along a quartic curve, or a double cover of $\mathbb{P}(1,1,4)$ branched along a degree 8 curve not passing through the vertex $[0: 0: 1]$. In the degree one case, $X_{\infty}$ is either a double cover of $\mathbb{P}(1,1,2)$ branched along the point $[0: 0: 1]$ and a sextic, or a double cover of $\mathbb{P}(1,2,9)$ branched along the point $[0: 1: 0]$ and a degree 18 curve not passing through the vertex $[0: 0: 1]$.

Proof. We first treat the case of degree one. The proof of the degree two case is essentially the same and we will add some remarks later. Denote by $Y_{i}$ the quotient of $X_{i}$ by $\sigma_{i}$, so the quotient $Y_{\infty}=X_{\infty} / \sigma_{\infty}$ is the Gromov-Hausdorff limit of $Y_{i}$ 's. For each integer $m$ we have an orthogonal decomposition $H^{0}\left(X_{i},-m K_{X_{i}}\right)=$ $V_{i} \oplus W_{i}$ with $V_{i}$ being the +1 eigenspace and $W_{i}$ the -1 eigenspace. Then we have a corresponding decomposition $H^{0}\left(X_{\infty},-m K_{X_{\infty}}\right)=V_{\infty} \oplus W_{\infty}$ on $X_{\infty}$. Now, by constructing orthonormal $\sigma_{\infty}$-invariant sections of $-k K_{X_{\infty}}$ for some $k$ large divisible, one can show that there is a well-defined map $\iota_{\infty}: X_{\infty} \rightarrow \mathbb{P}^{*}\left(V_{\infty}\right)$, which induces a projective embedding of $Y_{\infty}$. By an adaption of the Hörmander technique $(70,23)$, this implies that the orthonormal $\sigma_{i}$-invariant sections of $-k K_{X_{i}}$ (equivalent to sections of $-l K_{Y_{i}}$ for some integer $l$ ) define an embedding (Tian's embedding) of $Y_{i}$ into $\mathbb{P}^{*}\left(V_{i}\right)$ for $i$ sufficiently large. Moreover, we may assume $Y_{i}$ converges to $Y_{\infty}$ as normal varieties in $\mathbb{P}^{N}$ for some integer $N$. Since $Y_{i}$ 's are all isomorphic to $\mathbb{P}(1,1,2)$, we see that $Y_{\infty}$ is $\mathbb{Q}$-Gorenstein smoothable, and there is a partial $\mathbb{Q}$-Gorenstein smoothing of $Y_{\infty}$ to $\mathbb{P}(1,1,2)$.

Claim 5.3. $Y_{\infty}$ is isomorphic to $\mathbb{P}(1,2,9)$.

Since the degree is preserved in the limit, we have $K_{Y_{\infty}}^{2}=8$ and thus we may apply [30. Notice the full proof in [30] relies on the classification theorem of AlexeevNikulin [3], but in our case we only need the more elementary part [29], without 
use of [3. So we know $Y_{\infty}$ is either a toric log Del Pezzo surface $\mathbb{P}\left(a^{2}, b^{2}, 2 c^{2}\right)$ with $a^{2}+b^{2}+2 c^{2}=4 a b c$ or its partial smoothings. Since the orbifold structure group of $X_{\infty}$ always has order less than 12 , the order of all the orbifold structure groups of $Y_{\infty}$ must be less or equal to 22 . Then, by an easy investigation of the above Markov equation, we see that $Y_{\infty}$ must have two singularities, one of type $A_{1}$ and one of type $\frac{1}{9}(1,2)$. It could be possible that $Y_{\infty}$ is a partial smoothing of $\mathbb{P}\left(9, b^{2}, 2 c^{2}\right)$, but we claim then it must be $\mathbb{P}(1,2,9)$. For this we need to go back to the proof in [29]. For the minimal resolution $\pi: \widetilde{Y}_{\infty} \rightarrow Y_{\infty}$, let $n$ be the largest number such that there is a birational morphism $\mu_{n}$ from $\widetilde{Y}_{\infty}$ to the $n$-th Hirzebruch surface $\mathbb{F}_{n}$. Let $B^{\prime}$ be the proper transform of the negative section $B$ in $\mathbb{F}_{n}$, and let $p: \widetilde{Y}_{\infty} \rightarrow \mathbb{P}^{1}$ be the composition of $\mu_{n}$ with the projection map on $\mathbb{F}_{n}$. Then by a theorem of Manetti [47, Theorem 11] (see also [29, Theorem 5.1]) we know that $n \geq 2$, and the exceptional locus $E$ of $\pi$ is the union of $B^{\prime}$ and the components of degenerate fibers of $p$ with self-intersection at most -2 ; furthermore, each degenerate fiber of $p$ contains a unique -1 curve. Moreover, by the proof of Theorem 18 in 47] (see laso Theorem 5.7 in [29]), there are only two possible types for the dual diagram of the degenerate fiber: one type is that two strings of curves of self-intersection at most -2 joined by a $(-1)$-curve, and the other type is that we join a string of $(-2)$-curves though a $(-1)$ curve to the middle of a string of curves of self-intersection at most -2. In our case we know $Y_{\infty}$ has exactly one $A_{1}$ and one $\frac{1}{9}(1,2)$ singularity. By general theory on the resolution of cyclic quotient singularities we know $E$ is the disjoint union of a (-2)-curve and a string of a (-2)-curve and a (-5)-curve. Then one easily sees that the only possibility is that there is exactly one degenerate fiber of $\widetilde{Y}_{\infty}$ which consists of a string of (-2)-(-1)-(-2)-curve, and one of the (-2)-curves in the string intersects the horizontal section $B^{\prime}$ which is a $(-5)$-curve. Clearly $\widetilde{Y}_{\infty}$ is then a toric blown-up of $\mathbb{F}_{5}$ and then $Y_{\infty}$ is toric which must be $\mathbb{P}(1,2,9)$. This completes the proof of the claim.

The degree of the branched locus follows from the Hurwitz formula for coverings. The degree 18 curve can not pass through the point $[0: 0: 1]$, for otherwise the equation would be $a_{0} x_{3} f_{9}\left(x_{1}, x_{2}\right)+a_{1} f_{18}\left(x_{1}, x_{2}\right)=0$. Then by Lemma 5.6 below the singularity on the branched cover is not quotient, so can not be $X_{\infty}$ by Theorem 2.1. This finishes the proof of Theorem 5.2 for degree one case.

In the degree two case we can follow exactly the same arguments, noticing that, in this case, $Y_{\infty}$ must have degree equal to 9 and that the associated Markov type equation to be satisfied is now $a^{2}+b^{2}+c^{2}=3 a b c$ (corresponding to the weighted projective space $\left.\mathbb{P}\left(a^{2}, b^{2}, c^{2}\right)\right)$. Thus it follows, by inspection as above, that the only possibility is that $Y_{\infty}=\mathbb{P}(1,1,4)$ (since the standard projective plane is the only $\mathbb{Q}$-Gorenstein smoothing of the above weighted projective space).

Noticing that in the above situation our double cover can be realized as a hypersurface in a one dimensional higher weighted projective space, in terms of equations we have the following:

Corollary 5.4. In degree one case $X_{\infty}$ is either a sextic hypersurface in $\mathbb{P}(1,1,2,3)$ of the form $x_{4}^{2}=f_{6}\left(x_{1}, x_{2}, x_{3}\right)$, or a degree 18 hypersurface in $\mathbb{P}(1,2,9,9)$ of the form $x_{3}^{2}+x_{4}^{2}=f_{18}\left(x_{1}, x_{2}\right)$.

Corollary 5.5. In degree two case $X_{\infty}$ is either a quartic hypersurface in $\mathbb{P}(1,1,1,2)$ of the form $x_{4}^{2}=f_{4}\left(x_{1}, x_{2}, x_{3}\right)$ or an octic hypersurface in $\mathbb{P}(1,1,4,4)$ of the form $x_{3}^{2}+x_{4}^{2}=f_{8}\left(x_{1}, x_{2}\right)$.

Lemma 5.6. Suppose $f$ is a polynomial and the surface $w^{2}=f(x, y)$ in $\mathbb{C}^{3}$ or its $\mathbb{Z} / 2 \mathbb{Z}$ quotient by $(x, y, w) \mapsto(-x,-y,-w)$ has a quotient singularity at the origin, then $f$ must contain a monomial with degree at most three. 
Proof. If the singularity is a quotient singularity, then singularity $w^{2}=f(x, y)$ in $\mathbb{C}^{3}$ is canonical since the finite map does not have branch divisor. Then the statement follows from a criterion of canonicity in terms of Newton polygon (cf. e.g., [35, Corollary 1.7]).

The idea of using involutions to study $X_{\infty}$ was previously used in [70, where some partial results were claimed. For example, in Proposition 6.1 of [70], it was stated that in degree two case $X_{\infty}$ can have at most $\frac{1}{4}(1,1)$ singularities besides canonical singularities and $\left|-2 K_{X_{\infty}}\right|$ is base point free. This agrees with the above result. But, as one can see from the following example, the claims in Proposition 6.2 of [70] that in degree one case $X_{\infty}$ can have at most one non-canonical singularity and $\mid-2 K_{X_{\infty} \mid}$ is base point free, are both incorrect.

Now we show explicit examples of Kähler-Einstein log Del Pezzo surfaces with non-canonical singularities in both degree one and two 4 . In the next two subsections it will be proved that both are parametrized in the moduli spaces.

Example 5.7. Let $X_{2}^{T}$ be the quotient of $\mathbb{P}^{1} \times \mathbb{P}^{1}$ by the action of $\mathbb{Z} / 4 \mathbb{Z}$, where the generator $\xi$ of $\mathbb{Z} / 4 \mathbb{Z}$ acts by $\xi .\left(\left[z_{1}: z_{2}\right],\left[w_{1}: w_{2}\right]\right)=\left(\left[\sqrt{-1} z_{1}: z_{2}\right],\left[-\sqrt{-1} w_{1}: w_{2}\right]\right)$. Then it is easy to see that $X_{2}^{T}$ is a degree two log Del Pezzo surface, with two $A_{3}$ singularities and two $\frac{1}{4}(1,1)$ singularities. The standard product of round metrics on $\mathbb{P}^{1} \times \mathbb{P}^{1}$ descends to a Kähler-Einstein metric on $X_{2}^{T}$. The space $H^{0}\left(X_{2}^{T},-K_{X_{2}^{T}}\right)$ is spanned by the sections $z_{1}^{2} w_{1}^{2}, z_{2}^{2} w_{2}^{2}$, and $z_{1} z_{2} w_{1} w_{2}$. So a generic divisor in $\left|-K_{X_{2}^{T}}\right|$ is given by the union of two curves $z_{1} w_{1}+a z_{2} w_{2}=0$ and $z_{1} w_{1}+b z_{2} w_{2}=0$ for $a \neq b$, and is thus reducible. The space $H^{0}\left(X_{2}^{T},-2 K_{X_{2}^{T}}\right)$ is spanned by sections $z_{1}^{4} w_{1}^{4}, z_{2}^{4} w_{2}^{4}, z_{1}^{2} z_{2}^{2} w_{1}^{2} w_{2}^{2}, z_{1}^{3} z_{2} w_{1}^{3} w_{2}, z_{1} z_{2}^{3} w_{1} w_{2}^{3}, z_{1}^{4} w_{2}^{4}, z_{2}^{4} w_{1}^{4}$. The subspace $U$ spanned by the first five sections is generated by $H^{0}\left(X_{2}^{T},-K_{X_{2}^{T}}\right)$. The involution $\sigma$ maps $\left(\left[z_{1}: z_{2}\right],\left[w_{1}: w_{2}\right]\right)$ to $\left(\left[w_{1}: w_{2}\right],\left[z_{1}: z_{2}\right]\right)$. The +1 eigenspace $V_{1}$ is still six dimensional, spanned by $U$ and the element $z_{1}^{4} w_{2}^{4}+z_{2}^{4} w_{1}^{4}$. It is easy to see that the image of $X$ under the projection to $V$ is the cone over the rational normal curve of degree 4 , i.e. $\mathbb{P}(1,1,4)$. The branch locus is defined by $z_{1}^{4} w_{2}^{4}=z_{2}^{4} w_{1}^{4}$, with singularies exactly at the two $A_{3}$ singularities. We can also see directly that $X_{2}^{T}$ is the hypersurface in $\mathbb{P}(1,1,4,4)$ defined by $x_{1}^{4} x_{2}^{4}=x_{3} x_{4}$. The map is given by

$$
\left(\left[z_{1}: z_{2}\right],\left[w_{1}: w_{2}\right]\right) \mapsto\left(z_{1} w_{1}, z_{2} w_{2}, z_{1}^{4} w_{2}^{4}, z_{2}^{4} w_{1}^{4}\right) .
$$

Make a change of variable $x_{3}^{\prime}=x_{3}+x_{4}$ and $x_{4}^{\prime}=x_{3}-x_{4}$, then the projection to the $\left(x_{1}, x_{2}, x_{3}^{\prime}\right)$ plane realizes $X_{2}^{T}$ as a double cover of $\mathbb{P}(1,1,4)$.

Example 5.8. Let $X_{1}^{T}$ be the example studied in Section 3. It is a toric degree one Kähler-Einstein log Del Pezzo surface with one $A_{8}$ singularity and two $\frac{1}{9}(1,2)$ singularities. It can be viewed as a hypersurface in $\mathbb{P}(1,2,9,9)$ given by the equation $x_{3} x_{4}=x_{2}^{9}$. The embedding is defined by

$$
\left[z_{1}: z_{2}: z_{3}\right] \mapsto\left[z_{1}: z_{2} z_{3}: z_{2}^{9}: z_{3}^{9}\right] .
$$

The projection map $\mathbb{P}(1,2,9,9) \rightarrow \mathbb{P}(1,2,9)$ sending $\left[x_{1}: x_{2}: x_{3}: x_{4}\right]$ to $\left[x_{1}: x_{2}:\right.$ $\left.x_{3}+x_{4}\right]$ realizes $X_{1}^{T}$ as the double cover of $\mathbb{P}(1,2,9)$, branched along the rational curve $x_{2}^{9}=x_{3}^{2}$. On $X_{1}^{T}$ the holomorphic involution $\sigma$ simply exchanges $z_{2}$ with $z_{3}$. One can see the pluri-anti-canonial linear systems on $X_{1}^{T} \cdot H^{0}\left(X_{1}^{T},-K_{X_{1}^{T}}\right)$ is spanned by $z_{1}^{3}$ and $z_{1} z_{2} z_{3}$, so it has a fixed component $z_{1}=0 . H^{0}\left(X_{1}^{T},-2 K_{X_{1}^{T}}\right)$ is spanned by $z_{1}^{6}, z_{1}^{4} z_{2} z_{3}, z_{1}^{2} z_{2}^{2} z_{3}^{2}, z_{2}^{3} z_{3}^{3}$, so it has two base points $[0: 1: 0]$ and $[0: 0: 1]$. We will show below that $X_{1}^{T}$ is the Gromov-Hausdorff limit of a sequence of KählerEinstein degree one Del Pezzo surfaces. This implies that the Proposition 6.2 in

${ }^{4}$ We are indebted to A. Kasprzyk for discussions related to these examples [38]. 
70] is incorrect. Similarly it is easy to see that $\left|-3 K_{X_{1}^{T}}\right|$ is base point free. As before we have an eigenspace decomposition $H^{0}\left(X_{1}^{T},-m K_{X_{1}^{T}}\right)=V_{m} \oplus W_{m}$ for $\sigma$. Then $\left|V_{6}\right|$ is base point free, and it defines the embedding of $\mathbb{P}(1,2,9)$ into $\mathbb{P}^{15}$ by sections of $\mathcal{O}(18)$.

5.2. Degree two case. We first recall the moduli space constructed in 552. For a smooth Del Pezzo surface $X$ of degree 2 the anti-canonical map is a double covering to $\mathbb{P}^{2}$ branched along a smooth quartic curve $F_{4}$. The geometric invariant theory for quartic curves is well-understood (cf. [53]) as follows. (Note that Mukai's citation [52, 9.3] misses one case.)

Lemma 5.9 ([3] Theorem 2). For a quartic curve $F_{4}$ in $\mathbb{P}^{2}$ we have:

- $F_{4}$ is stable if and only if $F_{4}$ has only rational double points of type $A_{1}$ or $A_{2}$;

- $F_{4}$ is strictly polystable if and only if $F_{4}$ is one of the following: either a double conic or a union of two reduced conics that are tangential at two points and at least one is smooth (called cateye and ox in [33]).

It follows that the quotient $Q:=\mathbb{P}_{*}\left(\operatorname{Sym}^{4} \mathbb{C}^{3}\right)^{s s} / / P G L(3 ; \mathbb{C})$ parametrizes certain canonical log Del Pezzo surfaces of degree 2, away from the double conic. The stable curves parametrize surfaces with at worst $A_{1}$ or $A_{2}$ singularities, the double conic parametrize a non-normal surface with non orbifold singularities (note in fact that the variety of equation $t^{2}=\left(x^{2}+y^{2}+z^{2}\right)^{2}$ can be decomposed into irreducible factors $\left.\left(t+x^{2}+y^{2}+z^{2}\right)\left(t-x^{2}+y^{2}+z^{2}\right)=0\right)$, and the other polystable curves parametrize surfaces with exactly $2 A_{3}$ singularities. As in [52, we blow up the point corresponding to the double conic to obtain a new variety, denoted by $M_{2}$. Let $E$ be the exceptional divisor. Then, as in 64 , we know $E$ is isomorphic to the GIT moduli space $\mathbb{P}_{*}\left(S y m^{8} \mathbb{C}^{2}\right)^{s s} / / P G L(2 ; \mathbb{C})$, parametrizing binary octics $f_{8}(x, y)$. Moreover,

Theorem 5.10. $M_{2}$ is an analytic moduli space of log Del Pezzo surfaces of degree two. For any $[s] \notin E, X_{s}$ is the double cover of $\mathbb{P}^{2}$ branched along the polystable quartic defined by $[s]$, and for $[s] \in E, X_{s}$ is the double cover of $\mathbb{P}(1,1,4)$ (i.e. the cone over the rational normal curve in $\mathbb{P}^{5}$ ) branched along the hyperelliptic curve $z_{3}^{2}=f_{8}\left(z_{1}, z_{2}\right)$, where $f_{8}$ is the polystable binary octic defined by $[s]$.

The proof uses some ideas of 64 as written in [52, but note that the proof in 64 is incomplete about the existence of moduli algebraic stack nor the blow up is its coarse moduli scheme, since no family has been constructed. The argument in 64 is curve-wise and only verifies the properness criterion formally.

Proof. Let $\mathrm{H}_{4}$ be the Hilbert scheme of quartics in $\mathbb{P}^{2}$, and fix a non-degenerate conic $C=\{q=0\}$. We identify the automorphism group of $C$ with $P G L(2 ; \mathbb{C})$ (The notation $P G L(2 ; \mathbb{C})$ only appears in this context of this proof, so should not be confusing). Denote by $\Psi$ the (9-dimensional) $P G L(2 ; \mathbb{C})$-invariant subspace of $H^{0}\left(\mathbb{P}^{2}, \mathcal{O}(4)\right)$ that corresponds to $H^{0}\left(C,\left.\mathcal{O}(4)\right|_{C}\right)$. Take an affine space $\mathbb{A} \simeq \mathbb{C}^{9}$ in $H_{4}$ which represents $\left\{q^{2}+f_{4}(x, y, z)\right\}$ for all quartics $f_{4} \in \Psi$. From the construction, this gives a Luna étale slice. Note that the blow up $\mathbb{B}$ of $\mathbb{A}$ at 0 is a closed subvariety of $\mathbb{A} \times \mathbb{P}_{*}(\mathbb{A})$, and let $\mathbb{E}$ be its exceptional divisor. Let $\mathcal{B} \subset \mathbb{A} \times(\mathbb{A} \backslash\{0\})$ be the cone over $\mathbb{B}$, and $\mathcal{E}=\{0\} \times(\mathbb{A} \backslash\{0\})$ be the cone over $\mathbb{E}$. For each point $(a, b) \in \mathcal{B}$, we can associate the curve $q^{2}+b=0$ in $\mathbb{P}^{2}$. These form a flat projective family $\mathcal{Q}$ over $\mathcal{B}$.

On the other hand, consider the trivial family of $\left(\mathbb{P}^{2}, C\right)$ over $\mathcal{B}$. We blow up $C \times \mathcal{E}$ and contract the strict transform of $\mathbb{P}^{2} \times \mathcal{E}$. It is possible because $\mathcal{E}$ is a Cartier divisor in $\mathcal{B}$ and the classical degeneration (deformation to the normal cone 
of $C$ ) of $\mathbb{P}^{2}$ to $\mathbb{P}(1,1,4)$ over a smooth curve is constructed in the same way, so we can do it locally and glue the contraction morphism. Denote the family constructed in this way by $\mathcal{P} \rightarrow \mathcal{B}$. The generic fibers are $\mathbb{P}^{2}$ and special fibers (those over $\mathcal{E}$ ) are $\mathbb{P}(1,1,4)$. We also obtain a natural family of conics $C_{\mathcal{P}} \subset \mathcal{P}$ over $\mathcal{B}$.

All the above process is $P G L(2 ; \mathbb{C}) \times \mathbb{C}^{*}$-equivariant. Thus we can construct $P G L(2 ; \mathbb{C})$-invariant complement of $\mathbb{C} q^{2}$ in $H^{0}\left(\mathcal{P}_{u}, \mathcal{O}\left(2 C_{\mathcal{P}}\right)\right)(u \in \mathcal{B})$ in a continuous way, and extend the family of quartics $\left.\mathcal{Q}\right|_{(\mathcal{B} \backslash \mathcal{E})}$ to the whole $\mathcal{B}$. We denote the new total space by $\mathcal{D}$. Notice that over $\mathbb{E}$ this is a family of binary octics. Then construct $\mathcal{S}$ as the double of $\mathcal{P}$ branched along $\mathcal{D}$. As everything is again $P G L(2 ; \mathbb{C}) \times \mathbb{C}^{*}$ equivariant, we can first divide by $\mathbb{C}^{*}$ and obtain a $\mathbb{Q}$-Gorenstein flat family $S$ of degree two log Del Pezzo surfaces over $\mathbb{B}$.

There is still an action of $P G L(2 ; \mathbb{C})$ on $\mathbb{B}$. We consider GIT with respect to this action and with $P G L(2 ; \mathbb{C})$-linearized line bundle $\mathcal{O}_{\mathbb{B}}(-\mathbb{E})$. The natural morphism $\mathbb{B}^{s s} / / P G L(2 ; \mathbb{C}) \rightarrow \mathbb{A} / / P G L(2 ; \mathbb{C})$ is an isomorphic away from $\mathbb{E} \subset B$ and $0 \in \mathbb{A}$. So this is a blow up with exceptional divisor $\mathbb{E}^{s s} / / P G L(2 ; \mathbb{C})$. By the local picture of GIT ([64, Prop 5.1]), we can see that $\mathbb{A} / / P G L(2 ; \mathbb{C}) \rightarrow H_{4}^{s s} / / P G L(3 ; \mathbb{C})$ is étale (or in differential geometric language, local bi-holomorphism) around 0 . This follows completely the same way as in [64, Prop 5.1] or the proof of famous Luna étale slice theorem. Hence, the blow up $\mathbb{B}^{s s} / / P G L(2 ; \mathbb{C}) \rightarrow \mathbb{A} / / P G L(2 ; \mathbb{C})$ induces blow up $M_{2}$ of $H_{4} / / P G L(3 ; \mathbb{C})$.

To see that $M_{2}$ is an analytic moduli space for degree two log Del Pezzo surfaces, we only need to check the item (2) in the definition. For this, one simply notices that, by construction, for any $[s] \in M_{2}$ there is a Luna's slice $V$ in $H_{4}$ or in $\mathbb{B}$ (depending on whether $[s]$ is in $E$ or not). Then by versality there is an $\operatorname{Aut}\left(X_{s}\right)$ equivariant analytic map $\Psi_{U}$ from a small analytic neighborhood $U=V / / \operatorname{Aut}\left(X_{s}\right)$ of $[s]$ to the GIT quotient $\operatorname{Kur}\left(X_{s}\right) / / \operatorname{Aut}\left(X_{s}\right)$ so that $\Phi_{U}^{-1}(0)=0$. Then it follows that $\Psi_{U}$ is a finite map onto an open neighborhood of 0 .

In terms of étale topology one can also directly check the versality by going through our construction. We only need to check our $\left(H_{4}^{s s} \backslash P G L(3 ; \mathbb{C}) q^{2}\right) \coprod \mathbb{B}^{s s}$ is versal in étale topology. That is, given a $\mathbb{Q}$-Gorenstein projective family $f: \mathcal{X} \rightarrow$ $S$ of our log del Pezzo surfaces of degree 2, there is a morphism $\tilde{S} \rightarrow\left(H_{4}^{s s} \backslash\right.$ $\left.P G L(3 ; \mathbb{C}) q^{2}\right) \coprod B^{s s}$ compatible with fibers where $\tilde{S} \rightarrow S$ is an étale cover. For this, we can first construct a degenerating family of $\mathbb{P}^{2}$ to $\mathbb{P}(1,1,4)$ over $S$ and from the $\mathbb{Q}$-Gorenstein deformation theory of $\mathbb{P}(1,1,4)$ (with 1 -dimensional smooth semi-universal deformation space) we know that the locus of $\mathbb{P}(1,1,4)$ should be a Cartier divisor so that we can convert the process to obtain a family of reduced quartics of $\mathbb{P}^{2}$. Thus we have a compatible morphism to $\left(H_{4}^{s s} \backslash P G L(3 ; \mathbb{C}) q^{2}\right) \coprod B^{s s}$ locally in étale topological sense.

Remark 5.11. In terms of algebro-geometric language, $M_{2}$ coarsely represents the algebraic stack $\mathcal{M}_{2}$ constructed by gluing together the quotient stacks $\left[\mathbb{B}^{\text {ss }} / P G L(2 ; \mathbb{C})\right]$ and $\left[\left(H_{4}^{s s} \backslash P G L(3 ; \mathbb{C}) q^{2}\right) / P G L(3 ; \mathbb{C})\right]$.

Remark 5.12. Replacing blow up and its cone as above by weighted blow up and its quasi-cone, the argument in [64] can be completed to prove that the blow up is a coarse moduli scheme of degree two K3 surfaces and its degenerations.

The proof of Theorem 1.1 follows from the fact that all smooth degree two Del Pezzo surfaces are parametrized by $M_{2}$ and by

Theorem 5.13. $M_{2}$ has property (KE).

Proof. By Theorem 5.2 there are two possibilities for $X \in M_{2}^{G H}$ : it is either a double cover of $\mathbb{P}^{2}$ branched along a quartic $f_{4}\left(x_{1}, x_{2}, x_{3}\right)=0$, or a double cover of $\mathbb{P}(1,1,4)$ branched along a hyperelliptic octic curve $x_{3}^{2}-f_{8}\left(x_{1}, x_{2}\right)=0$. It suffices 
to show $f_{4}$ and $f_{8}$ are polystable. For this we use Theorem 3.3 and Theorem 3.4 When applying Theorem 3.4 in the first case we choose $S=\mathbb{P}_{*}\left(\operatorname{Sym}^{4}\left(\mathbb{C}^{3}\right)\right)$; in the second case we choose $S=\mathbb{P}_{*}\left(\operatorname{Sym}^{8}\left(\mathbb{C}^{2}\right)\right)$. Note that both these parameter spaces have Picard rank one. Also recall the first paragraph of the proof of (4.2) which asserts that there is no pathological test configurations in the sense of 43 in this situation. Thus we can apply theorem 3.16 and conclude that all the points in this glued moduli are indeed GH limits.

So we also conclude that $\mathcal{M}_{2}$ is a KE moduli stack. As it is immediately clear from the proof, the complex conjugation acts on $M_{2}$ by the natural anti-holomorphic involution.

Remark 5.14. In 70] it is conjectured that degenerations of Kähler-Einstein Del Pezzo surfaces should have canonical singularities. In this section we have seen that this conjecture is in general false, as all the surfaces parametrized by the exceptional divisor $E$ have exactly two non-canonical singularities of type $\frac{1}{4}(1,1)$. In general dimension one expects the compact moduli space of smoothable $\mathbb{Q}$-Fano varieties to have log terminal singularities, see [23]. This type of singularities also appear to be the worst singularities allowed for K-semistability of Fano varieties, see [58].

We finish this subsection by a discussion on the surfaces parametrized by the ox and cateyes, which will be used in our study of degree one case. These are defined by equations in $\mathbb{P}(1,1,1,2)$ parametrized by $\lambda=\left[\lambda_{1}: \lambda_{2}\right]$ in $\left(\mathbb{P}^{1} \backslash\{[1: 1]\}\right)$ which we denotes by $X_{2}^{\lambda}$. The equation of $X_{2}^{\lambda}$ is

$$
w^{2}=\left(\lambda_{1} z^{2}+x y\right)\left(\lambda_{2} z^{2}+x y\right) .
$$

It is clear that when we interchange $\lambda_{1}$ and $\lambda_{2}$ we get isomorphic surfaces. When $\lambda$ is $[1: 0]$ or $[0: 1]$, the branch locus is an ox and the surface $X_{2}^{\infty}=X_{2}^{\lambda}$ with exaclty two $A_{3}$ plus one $A_{1}$ singularities, otherwise the branch locus is a cateye and $X_{2}^{\lambda}$ with exactly two $A_{3}$ singularities. By Theorem 5.13 this family of surfaces all admit Kähler-Einstein metrics. As $\lambda$ tends to [1:1] these Kähler-Einstein surfaces converge to $X_{2}^{T}$, with the obvious Kähler-Einstein metric.

One can see that $X_{2}^{\infty}$ is a global quotient of $\mathbb{P}^{1} \times \mathbb{P}^{1}$, as follows. Consider the action of $\mathbb{Z} / 4 \mathbb{Z}$ on $\mathbb{P}^{1} \times \mathbb{P}^{1}$, where the generator $\xi$ acts by

$$
\xi \cdot\left(\left[z_{1}: z_{2}\right],\left[w_{1}: w_{2}\right]\right)=\left(\left[-w_{1}: w_{2}\right],\left[z_{1}: z_{2}\right]\right) .
$$

Then there are exactly four points with nontrivial isotropy. Let $Y$ be the quotient. Then the points $([0: 1],[0: 1])$ and $([1: 0],[1: 0])$ are $A_{3}$ singularities and ([1:0], $[0: 1])$ and $([0: 1],[1: 0])$ are $A_{1}$ singularities. One can see that the anti-canonical map $p$ from $Y$ to $\mathbb{P}^{2}$ is given by

$$
\left(\left[z_{1}: z_{2}\right],\left[w_{1}: w_{2}\right]\right) \mapsto\left(z_{1}^{2} w_{1}^{2}: z_{2}^{2} w_{2}^{2}: z_{1}^{2} w_{2}^{2}+z_{2}^{2} w_{1}^{2}\right),
$$

and the corresponding involution to the double covering structure is

$$
\sigma \cdot\left(\left[z_{1}: z_{2}\right],\left[w_{1}: w_{2}\right]\right)=\left(\left[w_{1}: w_{2}\right],\left[z_{1}: z_{2}\right]\right) .
$$

The branch locus is defined by $x y\left(z^{2}-4 x y\right)=0$ in $\mathbb{P}^{2}$, which is isomorphic to the ox. So $Y$ is exactly $X_{2}^{\infty}$, and it admits an explicit Kähler-Einstein metric.

Notice that $\mathbb{P}^{1} \times \mathbb{P}^{1}$ or $\mathbb{P}^{2}$ has no deformations, so their quotients by any finite group have no equisingular deformations. But clearly for $\lambda \neq[1: 0],[0: 1], X_{2}^{\lambda}$ has nontrivial equisingular deformations, so it can not be a global quotient of $\mathbb{P}^{2}$ or $\mathbb{P}^{1} \times \mathbb{P}^{1}$. 
5.2.1. Relation with moduli of curves. Naturally considering the associated branch locus for each double cover (i.e. the bi-anti-canonical map), we can regard our moduli $M_{2}$ as the GIT moduli of bi-canonically embedded Hilbert polystable genus 3 curves, which is constructed in [33. Indeed, by a direct comparison, the corresponding set of parametrized curves are the same. We have a 1-dimensional tacnodal curves and 5-dimensional hyperelliptic curves. They intersect at one point corresponding to the curve $z^{2}=x^{4} y^{4}$ in $\mathbb{P}(1,1,4)$. From this point of view, the proof that the moduli space is a blow up of the GIT moduli of plane quartics is given in [6] due to David Hyeon. Our proof recovers this result, modulo the criterion of the Hilbert stability.

Thus a natural question would be the corresponding "Del Pezzo surface modular interpretation" for the flipped contraction which contracts the tacnodal locus in the paper [33. In general, we can ask:

Question 5.15. What are the modular interpretations via log Del Pezzo surfaces for each step of the Hassett-Keel program in [33] In addition, are there also stability interpretations for them?

5.3. Degree one case. From Section 2 we know that for any $X \in M_{1}^{G H}$, there are only three possible types for the non-canonical singularities. Moreover, we have:

Lemma 5.16. The canonical singularities in $X \in M_{1}^{G H}$ are either $A_{1}, \cdots, A_{8}$ or $D_{4}$.

Proof. This follows from Theorem 2.7 and the Noether formula for singular surfaces [[30], Proposition 2.6]

$$
\rho(X)+K_{X}^{2}+\sum_{P \in \operatorname{Sing}(X)} \mu_{P}=12 \chi\left(\mathcal{O}_{X}\right)-2,
$$

where $\rho(X)$ is the Picard rank of $X$ and $\mu_{P}$ denotes the Milnor number. Notice that $\chi\left(\mathcal{O}_{X}\right)=1$ by the Kodaira vanishing theorem and that the Milnor number of an $A_{k}, D_{k}$ or $E_{k}$ singularity is $k$.

We mention that, by using the Kähler-Ricci flow and calculating certain $\alpha$ invariant, it has been proved in [77, 16] that a degree 1 log Del Pezzo surface with only $A_{n}$ singularities admits a Kähler-Einstein metric, if $n \leq 6$.

5.3.1. First step: GIT. By Corollary 5.4 a Gromov-Hausdorff limit in degree one is either a double cover of $\mathbb{P}(1,1,2)$ branched along a sextic or a double cover of $\mathbb{P}(1,2,9)$ branched along a degree 18 curve. As the first step, we will construct a moduli space of surfaces that are double cover of $\mathbb{P}(1,1,2)$ branched along a sextic that does not pass through $[0: 0: 1]$. These surfaces have equations $w^{2}=$ $F(x, y, z) \subset \mathbb{P}(1,1,2,3)$, where $F$ contains a nonzero term $z^{3}$.

Although the automorphism group of $\mathbb{P}(1,1,2)$ in non-reductive, we can construct a compact moduli space of such sextics in $\mathbb{P}(1,1,2)$ which are polystable in appropriate GIT sense, following [64]. Instead of the honest automorphism group $\operatorname{Aut}(\mathbb{P}(1,1,2))$, we consider the action of $S L(2 ; \mathbb{C}) \ltimes H^{0}\left(\mathbb{P}^{1}, \mathcal{O}(2)\right)$ which is a finite cover of $\operatorname{Aut}(\mathbb{P}(1,1,2))$ and a subgroup of $\operatorname{Aut}(\mathbb{P}(1,1,2), \mathcal{O}(2))$ (i.e. it also acts on the linearization). First we fix the translation action of $H^{0}\left(\mathbb{P}^{1}, \mathcal{O}(2)\right)$ by requiring the vanishing of the coefficient of $z^{2}$. Thus we only need to consider surfaces of the form

$$
w^{2}=z^{3}+f_{4}(x, y) z+f_{6}(x, y) .
$$

Then, by dividing out by the natural $\mathbb{C}^{*}$-action on $f_{4}$ and $f_{6}$ with weights 4,6 repectively, we obtain a weighted projective space $\mathbb{P}_{s}:=\mathbb{P}(2,2,2,2,2,3,3,3,3,3,3,3)$ as 
a parameter space. What is left is the action of $S L(2 ; \mathbb{C})$ in the two variables $x, y$. Thus we get a GIT quotient

$$
M_{1}^{\prime}:=\mathbb{P}_{s}^{s s} / / S L(2 ; \mathbb{C})
$$

as a moduli space. This is similar to 64, where the GIT of degree 12 curves in $\mathbb{P}(1,1,4)$ was studied. We have the following classification of singularities for polystable locus (compare 64, Theorem 4.3):

Lemma 5.17. With respect to the GIT stability of the above $S L(2 ; \mathbb{C})$-action, our surface $\left[w^{2}=z^{3}+z f_{4}(x, y)+f_{6}(x, y) \subset \mathbb{P}(1,1,2,3)\right]$ is:

(1) stable if and only if it contains at worst $A_{k}$ singularities;

(2) strictly polystable if and only if it contains exactly two $D_{4}$ singularities or $S L(2 ; \mathbb{C})$-equivalent to $p_{0}:=\left[-\frac{1}{3}\left(x^{2}+y^{2}\right)^{2}: \frac{2}{27}\left(x^{2}+y^{2}\right)^{3}\right]$ in $\mathbb{P}_{s}$ (in this case it is non-normal).

Proof. By the numerical criterion, a point $f=\left[f_{4}: f_{6}\right]$ is unstable if and only if there is a point $u \in \mathbb{P}^{1}(x, y)$ such that $f_{4}$ and $f_{6}$ has multiplicity bigger than two and three at $u$ respectively. Without loss of generality, we may assume $u=[1: 0]$, so that $y^{3}$ divides $f_{4}$ and $y^{4}$ divides $f_{6}$. Then it is easy to see that the corresponding sextic has a triple point at $u$, with unibranch (i.e. a unique tangent line). So the surface $X_{f}$ has an $E_{k}$ or worse singularity. Conversely if $X_{f}$ has a singularity of type $E_{k}$ or worse, then by multiplying by an element in $S L(2 ; \mathbb{C})$ we may assume the singularity is of the form $\left[1: 0: z_{0}\right] \in \mathbb{P}(1,1,2)$. In the affine chart where $x \neq 0$, the sextic is of the form $z^{3}+z f_{4}(1, y)+f_{6}(1, y)$. It is easy to see that the only triple point must have $y=z=0$. Then it follows that $\left[f_{4}: f_{6}\right]$ is unstable. Similarly, it is easy to see that $X_{f}$ is stable if and only if it contains at worst $A_{k}$ singularities, i.e. the sextic contains at worst double points. If $X_{f}$ is polystable, then $\left[f_{4}: f_{6}\right]$ must be in the $S L(2 ; \mathbb{C})$ orbit of $\left[a x^{2} y^{2}: b x^{3} y^{3}\right]$ for some non zero $[a: b] \in \mathbb{P}(2,3)$. It is not hard to see that for $[a: b] \in \mathbb{P}(2,3)$ not equal to $[-1 / 3: 2 / 27], X_{f}$ has exactly two $D_{4}$ singularities.

Remark 5.18. We remark that, in the context of rational elliptic surfaces (which is the blow up of the base point of a complete anti-canonical system of degree 1 Del Pezzo surface), Miranda [51] also analyzed the equivalent GIT stability and constructed the corresponding compactified moduli variety which is isomorphic to our $M_{1}^{\prime}$.

5.3.2. Second step: Blow up. For the compatibility with later discussions, we replace characters $x, y, z, w$ by $x^{\prime}, y^{\prime}, z^{\prime}, w^{\prime}$ for the homogeneous coordinates for $\mathbb{P}(1,1,2,3)$. Recall that in the statement of Theorem 5.2 when the Gromov-Hausdorff limit is the double cover of $\mathbb{P}(1,1,2)$, the branch locus could pass the vertex. This corresponds to the $z^{\prime 3}$ term vanishing in the statement $F\left(x^{\prime}, y^{\prime}, z^{\prime}\right)$. By Lemma 5.6. it is easy to see that if we want the surface to have only quotient singularities, there must be a term of the form $z^{\prime 2} f_{2}\left(x^{\prime}, y^{\prime}\right)$ where $f_{2}$ must have rank at least one. On the other hand, for these surfaces, there is no obvious reason that they do not appear as the Gromov-Hausdorff limit of Kähler-Einstein surfaces. Indeed we have explicit examples of such surfaces which admit Kähler-Einstein metric. The first is a one dimensional family of degree one Kähler-Einstein log Del Pezzo surfaces which are Gorenstein except one whose $f_{2}$ is rank two.

Example 5.19. We consider a $\mathbb{Z} / 2 \mathbb{Z}$ action on the family of degree two surfaces $X_{2}^{\lambda}$ as studied in the end of Section [5.2. The action is given by $[x: y: z: w] \mapsto[x:$ $y:-z:-w]$. The fixed points are exactly the singularities of $X_{2}^{\lambda}$. One can check that for $\lambda \neq[1: 0],[0: 1]$, the quotient $X_{1}^{\lambda}$ is a degree one log Del Pezzo surface with exactly two $D_{4}$ singularities. It is interesting that these surfaces admit a $\mathbb{C}^{*}$ 
action and correspond exactly to the polystable points in $M_{1}^{\prime}$, except $p_{0}$. From the discussion in the end of Section 5.2 we see they all admit Kähler-Einstein metrics.

For the surface $X_{2}^{\infty}$ the action fixes also the $A_{1}$ singularity $[0: 0: 1: 0]$, so the quotient $X_{1}^{\infty}$ has two $D_{4}$ singularities and one $\frac{1}{4}(1,1)$ singularity. Denote the embedding $\mathbb{P}(1,1,2) \hookrightarrow \mathbb{P}^{3}$ by $\left[x^{\prime}: y^{\prime}: z^{\prime}\right] \mapsto\left[z^{\prime}: x^{\prime 2}: x^{\prime} y^{\prime}: y^{\prime 2}\right]$. Then the bianti-canonical map realizes $X_{1}^{\infty}$ as a double cover of $\mathbb{P}(1,1,2) \subset \mathbb{P}^{3}$ branched along the curve isomorphic to $z^{\prime 2} x^{\prime} y^{\prime}+x^{\prime 3} y^{\prime 3}$. Indeed, $\left|-2 K_{X_{1}^{\infty}}\right|=\left|-2 K_{X_{2}^{\infty}}\right|^{\mathbb{Z} / 2 \mathbb{Z}}=$ $\left|\mathcal{O}_{\mathbb{P}(1,1,1,2)}(2)^{\mathbb{Z} / 2 \mathbb{Z}}\right|$ which is spanned by $x^{2}, x y, y^{2}, z^{2}$ so the branch locus is $x y z(z-$ $x y)$. The latter is isomorphic to the sextic described above.

So $X_{1}^{\infty}$ corresponds to the case that $f_{2}$ has rank two. Clearly $X_{1}^{\infty}$ admits a Kähler-Einstein metric, as a global quotient of $\mathbb{P}^{1} \times \mathbb{P}^{1}$.

The next example, which will be important in our further modification, is a degree one Kähler-Einstein log Del Pezzo surface which corresponds to $f_{2}$ being rank one.

Example 5.20. Consider the degree two surface $X_{2}^{\gamma_{0}}$ with $\gamma_{0}=[1:-1]$. It has two $A_{3}$ singularities, one at $[1: 0: 0: 0]$ and one at $[0: 1: 0: 0]$. Now consider the involution $\sigma: X_{2}^{\gamma_{0}} \rightarrow X_{2}^{\gamma_{0}}$ which sends $[x: y: z: w]$ to $[x:-y:-z:-w]$. Then $\sigma$ has two fixed points exactly at the two singularities. It is straightforward to check that the quotient, which we will denote by $X_{1}^{e}$ from now on, has one $A_{7}$ singularity and one $\frac{1}{8}(1,3)$ singularity. $\left|-2 K_{X_{1}^{e}}\right|$ is determined by the sections $\left\{x^{2}, y^{2}, y z, z^{2}\right\} \in H^{0}(\mathbb{P}(1,1,1,2), \mathcal{O}(2))$, and this defines a double covering map from $X_{1}^{e}$ to the quadric cone in $\mathbb{P}^{3}$. The corresponding involution $\sigma$ maps $[x: y: z$ : $w]$ to $[-x:-y:-z:-w]=[-x: y: z: w]$ (the identity holds on $X_{1}^{e}$ ). Then the fixed locus of $\sigma$ consists of the curve $w=0$ and the curve $x=0$. Denote again the embedding $\mathbb{P}(1,1,2) \hookrightarrow \mathbb{P}^{3}$ by $\left[x^{\prime}: y^{\prime}: z^{\prime}\right] \mapsto\left[z^{\prime}: x^{\prime 2}: x^{\prime} y^{\prime}: y^{\prime 2}\right]$. The branch locus in $\mathbb{P}(1,1,2)$ is isomorphic to the sextic $z^{\prime 2} x^{\prime 2}-z^{\prime} y^{\prime 4}=0$. So $X_{1}^{e}$ corresponds to that $f_{2}$ has rank one. Again $X_{1}^{e}$ admits a Kähler-Einstein metric by the discussion in the end of Section 5.2.

We have a refined classification than Corollary 5.4.

Lemma 5.21. Let $X_{\infty}$ be the Gromov-Hausdorff limit of a sequence of degree one Kähler-Einstein Del Pezzo surfaces. If it is a hypersurface in $\mathbb{P}(1,1,2,3)$ of the form $w^{2}=F_{6}(x, y, z)$, then either $F_{6}$ has a term $z^{3}$, or $F_{6}$ is equivalent to $z^{2}\left(x^{2}+y^{2}\right)+z g_{4}(x, y)+g_{6}(x, y)$ or $X_{0}$ is isomorphic to $X_{1}^{e}$.

Proof. Consider the case when $F_{6}$ contains no $z^{3}$ term. Then we claim the term $z^{2} f_{2}(x, y)$ must not vanish. Otherwise $F_{6}=z f_{4}(x, y)+f_{6}(x, y)$. Then in the affine chart $\{z \neq 0\}$ in $\mathbb{P}(1,1,2,3)$ we have equation $w^{2}=f_{4}(x, y)+f_{6}(x, y)$ then by the Lemma 5.6. $X_{\infty}$ has a non quotient singularity, so it can not be a Gromov-Hausdorff limit by Theorem 2.1. So up to equivalence we may assume the $z^{2}$ term in $F_{6}$ is of the form $z^{2}\left(x^{2}+y^{2}\right)$ or $z^{2} x^{2}$. In the former case we are done, so we assume the latter. Then we can write

$$
F_{6}(x, y, z)=z^{2} x^{2}+a z y^{4}+b z x f_{3}(x, y)+f_{6}(x, y) .
$$

Now if $a=0$, then again in the affine chart $\{z \neq 0\}$ we have equation $w^{2}=$ $x^{2}+b x f_{3}(x, y)+f_{6}(x, y)$. Then by a change of variable at $(0,0,0)$ we may assume it is locally equivalent to $w^{2}=x^{2}+a_{1} x y^{3}+a_{2} x y^{5}+a_{3} y^{6}$. It is easy to see this is either non-normal or has a $A_{i}$ singularity $i \geq 5$ at the origin. The corresponding singularity on $X_{0}$ is a $(\mathbb{Z} / 2 \mathbb{Z})$-quotient by the action $(x, y, w) \mapsto(-x,-y,-w)$. So $X_{0}$ is either non-normal or has an orbifold point of order at least 12 , thus it can not admit a Kähler-Einstein metric by Theorem 2.7 . 
So $a \neq 0$, then by a change of variables $y \mapsto y+c x$ and $z \mapsto z+g_{2}(x, y)$ we may assume

$$
F_{6}(x, y, z)=z^{2} x^{2}+z y^{4}+f_{6}(x, y) .
$$

$X_{1}^{e}$ is isomorphic to the surface defined by $w^{2}=z^{2} x^{2}+z y^{4}$. The one parameter subgroup $\lambda(t)=\left(t^{2}, t, 1, t^{2}\right)$ degenerates every surface defined by $(*)$ to $X_{1}^{e}$ as $t$ tends to zero. Since $X_{1}^{e}$ admits a Kähler-Einstein metric, it has vanishing Futaki invariant. By Theorem 3.3 we see $X_{\infty}$ must be isomorphic to $X_{1}^{e}$.

We first construct a moduli space for surfaces with $f_{2}$ being rank two, and we will show these surfaces are parametrized exactly by a weighted blow up of $M_{1}^{\prime}$ at $p_{0}$. The surfaces are defined by

$$
w^{\prime 2}=z^{\prime 2}\left(x^{\prime 2}+y^{\prime 2}\right)+z^{\prime} g_{4}\left(x^{\prime}, y^{\prime}\right)+g_{6}\left(x^{\prime}, y^{\prime}\right) .
$$

Similarly as before, by considering the translation $z^{\prime} \mapsto z^{\prime}+a_{2}\left(x^{\prime}, y^{\prime}\right)$ for certain quadric $a_{2}\left(x^{\prime}, y^{\prime}\right)$, we may assume $g_{4}$ lies in the space $T\left(x^{\prime}, y^{\prime}\right):=\mathbb{C}\left(x^{\prime}+i y^{\prime}\right)^{4} \oplus$ $\mathbb{C}\left(x^{\prime}-i y^{\prime}\right)^{4}$, which is the $S O(2 ; \mathbb{C})\left(\cong \mathbb{C}^{*}\right)$-invariant complement to the linear subspace of $\operatorname{Sym}^{4}\left(\mathbb{C} x^{\prime} \oplus \mathbb{C} y^{\prime}\right)$ consists of those divisible by $\left(x^{\prime 2}+y^{\prime 2}\right)$. In this way, we can obtain GIT quotient $\mathbb{P}_{e}^{s s} / / S O(2 ; \mathbb{C}):=\mathbb{P}(1,1,2,2,2,2,2,2,2)^{s s} / / S O(2 ; \mathbb{C})$ which parametrizes surfaces of the form (5.1). Here we need to specify the weight of $S O(2 ; \mathbb{C}) \cong \mathbb{C}^{*}$ on the linearization, and we choose the natural one, so the action corresponding to $\left(x^{\prime}+i y^{\prime}\right) \mapsto \mu\left(x^{\prime}+i y^{\prime}\right),\left(x^{\prime}-i y^{\prime}\right) \mapsto \mu^{-1}\left(x^{\prime}-i y^{\prime}\right)$ has weight

$$
(4,-4,6,4,2,0,-2,-4,-6),
$$

with respect to the basis consists of

$$
\left(x^{\prime}+i y^{\prime}\right)^{4},\left(x^{\prime}-i y^{\prime}\right)^{4},
$$

and

$$
\begin{aligned}
& \left(x^{\prime}+i y^{\prime}\right)^{6},\left(x^{\prime}+i y^{\prime}\right)^{5}\left(x^{\prime}-i y^{\prime}\right), \\
& \left(x^{\prime}+i y^{\prime}\right)^{4}\left(x^{\prime}-i y^{\prime}\right)^{2},\left(x^{\prime}+i y^{\prime}\right)^{3}\left(x^{\prime}-i y^{\prime}\right)^{3}, \\
& \left(x^{\prime}+i y^{\prime}\right)^{2}\left(x^{\prime}-i y^{\prime}\right)^{4},\left(x^{\prime}+i y^{\prime}\right)\left(x^{\prime}-i y^{\prime}\right)^{5},\left(x^{\prime}-i y^{\prime}\right)^{6} .
\end{aligned}
$$

Then we have the following.

Lemma 5.22. The GIT quotient $\mathbb{P}_{e}^{s s} / / S O(2 ; \mathbb{C})$ with respect to the action with weight (5.2) above parametrizes log Del Pezzo surfaces, i.e. a polystable sextic defined by $\left[g_{4}: g_{6}\right] \in P_{e}$ has only quotient singularities, or more precisely, the corresponding Del Pezzo surface has exactly one $\frac{1}{4}(1,1)$ singularity besides canonical singularities.

Proof. It is easy to check that if a sextic has the form $z^{\prime 2}\left(x^{\prime 2}+y^{\prime 2}\right)+z^{\prime}\left(a\left(x^{\prime}+\right.\right.$ $\left.\left.i y^{\prime}\right)^{4}+b\left(x^{\prime}-i y^{\prime}\right)^{4}\right)+g_{6}\left(x^{\prime}, y^{\prime}\right)$ with $a, b \neq 0$, then it has only double points away from the vertex. If $a=b=0$, then for it to be stable, it has at most double points, and for it to be polystable, it has exactly two $D_{4}$ singularities besides the vertex. If $a \neq 0$ and $b=0$, then, if it is stable, the sextic has at most double points, and if it is semistable, then it degenerates to $z^{\prime 2}\left(x^{\prime 2}+y^{\prime 2}\right)+a\left(x^{\prime}+i y^{\prime}\right)^{3}\left(x^{\prime}-i y^{\prime}\right)^{3}$, which has two $D_{4}$ singularities.

When we prove the moduli space we constructed in the end has property (KE) we need to show:

Lemma 5.23. A surface of the form (5.1) that admits a Kähler-Einstein metric must be GIT polystable with respect to the chosen linearization as above. 
Proof. This does not follow directly from the general Theorem 3.4, as the group $S O(2 ; \mathbb{C}) \cong \mathbb{C}^{*}$ has non trivial characters. But in our case this can be done by explicit analysis as follows. Notice that since $\mathbb{P}_{e}$ contains a point parametrizing a K-polystable log Del Pezzo surface (e.g. $X_{1}^{\infty}$ ), the CM line bundle must be isomorphic to $\mathcal{O}(k)$ for $k>0$. This follows from the proof of Theorem 3.4. $X_{1}^{\infty}$ corresponds to the vector $v=[0: 0: 0: 0: 0: 1: 0: 0: 0]$ in $\mathbb{P}_{e}$ with respect to the quasi-homogeneous coordinates as above. So the weight of the action on the CM line bundle must also be the natural one as above, for otherwise it is easy to see that $v$ is unstable.

The second step toward the construction of $M_{1}$ is to replace the point $\left[p_{0}\right] \in M_{1}^{\prime}$ (which corresponds to a non-normal surface) by the above GIT quotient.

Theorem 5.24. There is a blow up $M_{1}^{\prime \prime} \rightarrow M_{1}^{\prime}$ at $\left[p_{0}\right]$ (with a non-reduced ideal) so that $M_{1}^{\prime \prime}$ is an analytic moduli space for degree one log Del Pezzo surfaces. The exceptional divisor $E$ is isomorphic to $\mathbb{P}_{e}^{s s} / / S O(2 ; \mathbb{C})$. Moreover, a point $s \in M_{1}^{\prime \prime}$ parametrizes the polystable sextic hypersurface $X_{s}$ defined by it, and $s \in E$ if and only if the sextic passes through the vertex $[0: 0: 1]$.

Proof. Let $\tilde{\mathbb{A}} \simeq \operatorname{Sym}^{4}(\mathbb{C} x \oplus \mathbb{C} y) \oplus \operatorname{Sym}^{6}(\mathbb{C} x \oplus \mathbb{C} y)$ be the cone over $\mathbb{P}_{s}$. In the tangent space at the point $p_{0}=\left(-\frac{1}{3}\left(x^{2}+y^{2}\right)^{2}, \frac{2}{27}\left(x^{2}+y^{2}\right)^{3}\right)$, we take an $S O(2 ; \mathbb{C})$ invariant Luna étale slice $\mathbb{A}_{f}:=p_{0}+\left\{T(x, y) \oplus \operatorname{Sym}^{6}(\mathbb{C} x \oplus \mathbb{C} y)\right\}$ in $\tilde{\mathbb{A}}$. To include surfaces of the form (5.1), let $\mathbb{A}_{g}=T\left(x^{\prime}, y^{\prime}\right) \oplus S y m^{6}\left(\mathbb{C} x^{\prime} \oplus \mathbb{C} y^{\prime}\right)$, and we consider the family of surfaces over $\mathbb{A}_{g} \times \mathbb{C}^{*}$ where we associate $\left(g_{4}, g_{6}, t\right)$ the sextic

$$
t z^{\prime 3}+z^{\prime 2}\left(x^{\prime 2}+y^{\prime 2}\right)+z^{\prime} g_{4}\left(x^{\prime}, y^{\prime}\right)+g_{6}\left(x^{\prime}, y^{\prime}\right) \text {. }
$$

Making the change of variable

$$
x^{\prime}:=t x, y^{\prime}:=t y, z^{\prime}:=z-\frac{t}{3}\left(x^{2}+y^{2}\right),
$$

and

$$
\begin{gathered}
f_{4}(x, y)=-\frac{t^{2}}{3}\left(x^{2}+y^{2}\right)^{2}+t^{3} g_{4}(x, y) \\
f_{6}(x, y)=\frac{2 t^{3}}{27}\left(x^{2}+y^{2}\right)^{3}-\frac{t^{4}}{3}\left(x^{2}+y^{2}\right) g_{4}(x, y)+t^{5} g_{6}(x, y)
\end{gathered}
$$

the sextic in equation (5.3) is then transformed into the form

$$
t\left[z^{3}+f_{4}(x, y) z+f_{6}(x, y)\right] .
$$

Hence it corresponds to the point $\left[f_{4}(x, y): f_{6}(x, y)\right] \in \mathbb{A}_{f} \subseteq \mathbb{P}_{s}$. If we keep $g_{4}$ and $g_{6}$ fixed, and let $t$ tend to zero this converges exactly to the point $p_{0}$.

The equation (5.3) defines a family of sextics over the trivial $\mathbb{P}_{x^{\prime}, y^{\prime}, z^{\prime}}(1,1,2)$ bundle $\mathcal{P}^{\prime}$ over $\mathbb{A}_{g} \times \mathbb{C}^{*}$, and it extends obviously over $\mathbb{A}_{g} \times \mathbb{C}$, which is the cone over the blow up $\mathbb{B}_{g}$ of $\mathbb{A}_{g}$ at 0 . This family is invariant under $\mathbb{C}^{*}$ action $\lambda .\left(t, g_{4}, g_{6}\right):=$ $\left(\lambda^{-1} t, \lambda g_{4}, \lambda^{2} g_{6}\right)$, and thus descended to a family over $\mathbb{B}_{g}$. The above change of variables indeed defines an isomorphism $\Psi$ between $\mathcal{P}=\mathbb{P}_{x, y, z}(1,1,2) \times\left(\mathbb{A}_{f} \times \mathbb{C}^{*}\right)$, and induces a $\mathbb{C}^{*}$ action on $\mathbb{A}_{f}$. We decompose $\mathbb{A}_{f}$ as $\mathbb{A}_{f}=p_{0}+\left(L_{1} \oplus L_{2}\right)$, where

and

$$
\left.L_{1}:=\left\{\left(f_{4}(x, y),-\frac{1}{3}\left(x^{2}+y^{2}\right) f_{4}(x, y)\right)\right\} \mid f_{4} \in T_{(x, y)}\right\}
$$

$$
L_{2}:=\operatorname{Sym}^{6}(\mathbb{C} x \oplus \mathbb{C} y) \subset \mathbb{A}_{f} .
$$

Denote the associated ideals of $L_{i}+p_{0}$ in $\mathbb{A}_{f}$ by $I_{\left(L_{i}+p_{0}\right)}$. Then we define $\mathbb{B}_{f}$ to be the blow up of $\mathbb{A}_{f}$ at $I_{\left(L_{1}+p_{0}\right)}^{2}+I_{\left(L_{2}+p_{0}\right)}$. The exceptional divisor is isomorphic to $\mathbb{P}_{e}$. Then by pulling back by $\Psi$ we obtain a flat family of sextics over $\mathbb{B}_{f}$, and the exceptional divisor parametrizes sextics of the form (5.1). 
Similarly to the degree 2 case, we consider GIT of $\mathbb{B}_{f}$ with respect to the $S O(2 ; \mathbb{C})$-action, and get a certain blow up $\mathbb{B}_{f}^{s s} / / S O(2 ; \mathbb{C}) \rightarrow \mathbb{A}_{f} / / S O(2 ; \mathbb{C})$. This induces a blow up of $\mathbb{P}_{s} / / S L(2 ; \mathbb{C})$ at $\left[p_{0}\right]$, with exceptional divisor $E \cong \mathbb{P}_{e}^{s s} / / S O(2 ; \mathbb{C})$. We denote this by $M_{1}^{\prime \prime} \rightarrow M_{1}^{\prime}$.

From the construction, as in the previous section, $M_{1}^{\prime \prime}$ is an analytic moduli space and a coarse moduli of an algebraic stack which is constructed by gluing

$$
\left[\mathbb{B}_{f}^{s s} / S O(2 ; \mathbb{C})\right]
$$

naturally with

$$
\left[\left(\mathbb{P}_{s}^{s s} \backslash\left(P G L(2 ; \mathbb{C}) \cdot p_{0}\right)\right) / P G L(2 ; \mathbb{C})\right]
$$

in our context.

5.3.3. Construction of moduli: further modifications. We have a further refinement of Corollary 5.4 parallel to Lemma 5.21

Lemma 5.25. Let $X_{\infty}$ be the Gromov-Hausdorff limit of a sequence of degree one Kähler-Einstein Del Pezzo surfaces. Then $X_{\infty}$ is a sextic hypersurface in $\mathbb{P}(1,1,2,3)$ of the form $x_{4}^{2}=f_{6}\left(x_{1}, x_{2}, x_{3}\right)$, or isomorphic to the toric surface $X_{1}^{T}$.

Proof. By Theorem 5.2, we may assume $X_{\infty}$ is a degree 18 hypersurface in $\mathbb{P}(1,2,9,9)$ of the form $x_{4}^{2}=f_{18}\left(x_{1}, x_{2}, x_{3}\right)$ not passing through the point $[0: 0: 1]$. So we may assume $f_{18}\left(x_{1}, x_{2}, x_{3}\right)=x_{3}^{2}+g_{18}\left(x_{1}, x_{2}\right)$. If the term $x_{2}^{9}$ appears in $g_{18}$, then the one parameter subgroup $\Lambda$ acting with weight $(0,9,2,2)$ degenerates $x_{4}^{2}-f_{18}$ to $x_{4}^{2}-x_{3}^{2}-a x_{2}^{9}$. This induces a test configuration for $X_{\infty}$ with central fiber isomorphic to $X_{1}^{T}$. Since $X_{1}^{T}$ has vanishing Futaki invariant, and $X_{\infty}$ is K-polystable, we conclude that $X_{\infty}$ must be isomorphic to $X_{1}^{T}$. If $x_{2}^{9}$ does not appear in $g_{18}$, then the one parameter subgroup $\Lambda$ acting with weight $(0,0,1,1)$ degenerates $x_{4}^{2}-f_{18}\left(x_{1}, x_{2}, x_{3}\right)$ to $x_{4}^{2}-x_{3}^{2}$. Again this induces a test configuration for $X_{\infty}$ with central fiber the nonnormal hypersurface $Y$ defined by $x_{4}^{2}-x_{3}^{2}=0$. We claim this has zero Futaki invariant, thus contradicting the fact that $X_{\infty}$ is K-polystable. To see the claim, note that the Futaki invariant for a $\mathbb{C}^{*}$-action on a connected fixed component in the Hilbert scheme is constant. Since $X_{1}^{T}$ obviously degenerates to $Y$ and is fixed by the same $\Lambda$, we can compute the Futaki invariant on $X_{1}^{T}$, which is zero since it is Kähler-Einstein.

The analytic moduli space $M_{1}^{\prime \prime}$ constructed in the previous section does not have property $(\mathrm{KE})$, since it does not parametrize the two examples $X_{1}^{e}$ and $X_{1}^{T}$ which we are unable to show that they can not appear as a Gromov-Hausdorff limit. So we have to make a modification of $M_{1}^{\prime \prime}$. Now the only problem is to fit these two into $M_{1}^{\prime \prime}$. We first illustrate the phenomenon of modification of GIT by a simple example.

Example 5.26. Let $\mathbb{C}^{*}$ act linearly on $\mathbb{C}^{2}$ by $t .\left(z_{1}, z_{2}\right)=\left(t z_{1}, z_{2}\right)$. Then the quotient is isomorphic to $\mathbb{C}$, and the polystable locus are points on the line $\{0\} \times \mathbb{C}$. If we remove the origin $(0,0)$, then the quotient is again isomorphic to $\mathbb{C}$, but the polystable locus differs from the previous one in that the orbit of the origin is replaced by the punctured line $\mathbb{C}^{*} \times\{0\}$.

Our situation is very similar to this. We first investigate the $\mathbb{Q}$-Gorenstein deformation of $X_{1}^{T}$ studied in Section 3. Adopting the notation there, we have:

Lemma 5.27. A point $v=\left(v_{1}, v_{2}, v_{3}\right) \in \operatorname{Def}\left(X_{1}^{T}\right)$ is polystable under the action of $A u t^{0}\left(X_{1}^{T}\right)$ if and only if $v_{1}, v_{2}$ and $v_{3}$ are all non-zero or all zero, and $(0,0,0)$ is the only strictly polystable point. 
Proof. If $v_{1}=0$, then we can destabilize $v$ by the one-parameter subgroup $\lambda(t)=$ $\left(t^{-1}, 1\right)$. If $v_{2}=0$, then we can destabilize $v$ by the one-parameter subgroup $\left(1, t^{-1}\right)$. If $v_{3}=0$, then we can destabilize $v$ by the one-parameter subgroup $\left(t^{3}, t^{2}\right)$. If all the $v_{i}$ 's are non-zero, then for $\lambda(t)=\left(t^{a}, t^{b}\right)$ to destabilize $v$ we need $a-b \geq 0$, $-3 a+6 b \geq 0$, and $-3 a-3 b \geq 0$. It is easy to see that no non-trivial such pair $(a, b)$ exists.

To fill $X_{1}^{T}$ in our moduli, since we may locally identify $\operatorname{Kur}\left(X_{1}^{T}\right)$ with $\operatorname{Def}\left(X_{1}^{T}\right)$, and the $\left(\mathbb{C}^{*}\right)^{2}$-action on $\operatorname{Kur}\left(X_{1}^{T}\right)$ is compatible with the one on $\operatorname{Def}\left(X_{1}^{T}\right)$, it suffices to study the GIT on $\operatorname{Def}\left(X_{1}^{T}\right)$. By the above lemma, the stable points all represent canonical log Del Pezzo surfaces with at most a unique $A_{k}(k \leq 7)$ singularity, and the polystable point 0 represents $X_{1}^{T}$. The GIT quotient $Q$ is then smooth at $\left[X_{1}^{T}\right]$. The semistable orbit $\left(0, v_{2}, v_{3}\right)$ (where $0<\left|v_{2}\right|^{2}+\left|v_{3}\right|^{2} \ll 1$ ) represent a log Del Pezzo surface with a unique $A_{8}$ singularity. Since it is unique up to isomorphism by [26, we denote it by $X_{1}^{a}$. Due to Lemma 5.17 it has discrete automorphism group and it is parametrized by a point $u_{0}$ in $M_{1}^{\prime \prime} \backslash E$.

Consider the analytic subset $\operatorname{Kur}^{\prime}\left(X_{1}^{T}\right)$ of $\operatorname{Kur}\left(X_{1}^{T}\right)$ which represents only canonical $\log$ Del Pezzo surfaces, i.e. that consists of points with $v_{2} \neq 0$ and $v_{3} \neq 0$. Then the corresponding quotient $Q^{\prime}$ can be identified with the previous quotient $Q$, which identifies every stable orbit, except the orbit of $X_{1}^{a}$ is replaced by $X_{1}^{T}$. $Q^{\prime}$ can be viewed as the universal deformation space $X_{1}^{a}$. There is an analytic neighborhood $U$ of $u_{0}$, and an embedding $\iota: U \rightarrow Q^{\prime}=Q$ such that $\iota\left(u_{0}\right)=0$, and $u$ and $\iota(u)$ parametrize equivalent surfaces. In terms of stack language, the open embedding of stacks $\left[\left(\operatorname{Kur}\left(X_{1}^{T}\right) \backslash \operatorname{Kur}\left(X_{1}^{T}\right)^{\prime}\right) /\left(\mathbb{C}^{*}\right)^{2}\right] \hookrightarrow\left[\operatorname{Kur}\left(X_{1}^{T}\right) /\left(\mathbb{C}^{*}\right)^{2}\right]$ induces an isomorphism of the categorical moduli. Now we can simply define $M_{1}^{\prime \prime \prime}=M_{1}^{\prime \prime}$ as a variety and only change the surface parametrized by $u_{0}$ from $X_{1}^{a}$ to $X_{1}^{T}$. Then it is clear that $M_{1}^{\prime \prime \prime}$ is again an analytic moduli space of degree one log Del Pezzo surfaces. So this modification takes care of the point $X_{1}^{T}$.

Now we treat $X_{1}^{e}$ in a similar fashion. First notice that the linear system |$2 K_{X_{1}^{e}} \mid$ realizes $X_{1}^{e}$ as the double cover of $\mathbb{P}(1,1,2)$, thus $\operatorname{Aut}^{0}\left(X_{1}^{e}\right)$ is induced from $\operatorname{Aut}(\mathbb{P}(1,1,2))$. Then one sees that $\operatorname{Aut}^{0}\left(X_{1}^{e}\right) \cong \mathbb{C}^{*}$ corresponds to the scaling $\lambda(t)=\left(t^{2}, t, 1, t^{2}\right)$. By Lemma 3.9. we have

$$
\operatorname{Def}\left(X_{1}^{e}\right)=\operatorname{Def}^{\prime} \oplus \operatorname{Def}_{1} \oplus \operatorname{Def}_{2},
$$

where Def $^{\prime}$ corresponds to equisingular deformations, Def $_{1}$ corresponds to deformations of the local singularity at $[0: 0: 1: 0]$, and $\mathrm{Def}_{2}$ corresponds to deformations of the local singularity at $[1: 0: 0: 0]$. By applying again the Main Theorem of 47, it follows that Def $f_{1}$ is two dimensional and Def $_{2}$ is seven dimensional. Thus by dimension counting we must have $\operatorname{Def}^{\prime}=0$. We can write down a semi-universal deformation family:

$$
w^{2}=z^{2} x^{2}+z y^{4}+a_{1} z^{3}+a_{2} z^{2} y^{2}+\sum_{i=0}^{6} b_{i} x^{i} y^{6-j},
$$

In particular, note that we have $\operatorname{Aut}\left(X_{1}^{e}\right)$-invariant affine versal deformation space $\operatorname{Kur}\left(X_{1}^{e}\right)$ as claimed in the explanation after Lemma 3.9 and in this case, $\operatorname{Kur}\left(X_{1}^{e}\right)$ can be identified globally with the tangent space $\operatorname{Def} X_{1}^{e}$ so that $\left(a_{1}, a_{2}\right) \in \operatorname{Def}_{1}$ and $\left(b_{0}, \cdots, b_{6}\right) \in$ Def $_{2}$.

It is also easy to see the weights of the action is

$$
\lambda(t) .(a, b)=\left(t^{-4}, t^{-2}, t^{8}, t^{6}, \cdots, t^{2}\right) .
$$

So in the local GIT quotient by $\operatorname{Aut}\left(X_{1}^{e}\right)$ a point $(a, b)$ is stable if and only if $a \neq 0$ and $b \neq 0$ in which case $X_{a, b}$ has either a unique $A_{k}(k \leq 6)$ singularity or a $\frac{1}{4}(1,1)$ plus $A_{k}(k \leq 6)$ singularity. 
When we remove the subspace $\{0\} \oplus$ Def $_{2}$, every point becomes stable. In particular, the quotient of the subspace $(a, 0)$ with $a \neq 0$ is exactly a $\mathbb{P}^{1}$, which parametrizes surfaces in $M_{1}^{\prime \prime}$

$$
w^{2}=a_{1} z^{3}+z^{2} x^{2}+z y^{4}+a_{2} z^{2} y^{2},
$$

and intersects the exceptional divisor at one point corresponding to $a_{1}=0$. It is easy to see that $\lambda(t)$ degenerates all these surfaces to $X_{1}^{e}$ as $t$ tends to infinity, so they could not admit Kähler-Einstein metrics, and we need to remove them. Notice this family does not include the point corresponding to $X_{1}^{a}$, so we can make a further modification simultaneously as the previous one. When we add the the subspace $\{0\} \oplus \operatorname{Def}_{2}$, the point $(a, 0)$ with $a \neq 0$ become semistable and in the GIT quotient this is contracted to the point 0 . To be more precise we take the neighborhood $U$ in $\operatorname{Def}\left(X_{1}^{e}\right)$ consisting of points $(a, b)$ with ||$a|-1| \ll 1$ and $|b| \ll 1$, and the quotient $V$ by $\mathbb{C}^{*}$ gives rise to a tubular neighborhood of the $\mathbb{P}^{1}$ in $M_{1}^{\prime \prime}$. When we add the subspace $\{0\} \oplus \operatorname{Def}_{2}$ we have that $V$ gets mapped to a neighborhood of 0 in the local GIT, with $\mathbb{P}^{1}$ contracted to 0 .

As before the GIT on $\operatorname{Kur}\left(X_{1}^{e}\right)$ and on $\operatorname{Def}\left(X_{1}^{e}\right)$ are equivalent so this allows us to perform the contraction in an analytic neighborhood of the $\mathbb{P}^{1}$ inside $M_{1}^{\prime \prime \prime}$. We obtain a new analytic moduli space $M_{1}$, which enjoys the Moishezon property. Thus it has a natural structure of an algebraic space as well.

Theorem 1.1 in degree one case then follows from the theorem below.

Theorem 5.28. $M_{1}$ has property (KE).

Proof. The proof is very similar to Theorem 5.13. By Lemma 5.25 we only need to show that if a $X \in M_{1}^{G H}$ is a sextic hypersurface in $\mathbb{P}(1,1,2,3)$ defined by $w^{2}=f_{6}(x, y, z)$, then it is parametrized by some element in $M_{1}^{\prime \prime}$. If $f_{6}$ contains a term $a z^{3}$ with $a \neq 0$, then it is parametrized a point $u$ by $\mathbb{P}_{s}$. Then by Theorem 3.3 and Theorem 3.4, keeping in mind that $\mathbb{P}_{s}$ has Picard rank one, we conclude that $u$ is polystable under the $S L(2 ; \mathbb{C})$ action, thus $X$ is parametrized by a point $p$ in $M_{1}^{\prime}$. Then $X$ can not be isomorphic to $X_{1}^{T}$ or the $\mathbb{P}^{1}$ family above. So $X$ is parametrized by a point in $M_{1}$. If the term $z^{3}$ does not appear in $f_{6}$, then by Lemma [5.21] and Lemma [5.23 $X$ is either isomorphic to $X_{1}^{e}$ or is parametrized by a polystable point $u \in \mathbb{P}_{e}$. Again this point $u$ can not be on the $\mathbb{P}^{1}$ and this means that $u$ is in $M_{1}$.

We can construct a $\mathrm{KE}$ moduli stack $\mathcal{M}_{1}$ by gluing the previously constructed moduli stack with $\left[U / \operatorname{Aut}\left(X_{1}^{e}\right)\right]$ where $U$ is some open $\operatorname{Aut}\left(X_{1}^{e}\right)$-invariant neighborhood of $0 \in \operatorname{Kur}\left(X_{1}^{e}\right)$ (along $\left.\left[\left(U \backslash\left(\{0\} \oplus \operatorname{Def}_{2}\right)\right) / \operatorname{Aut}\left(X_{1}^{e}\right)\right]\right)$. Recall that in this case, we identified globally $\operatorname{Kur}\left(X_{1}^{e}\right)$ and $\operatorname{Def}\left(X_{1}^{e}\right)$. We can show that with a small enough $\operatorname{Aut}\left(X_{1}^{e}\right)$-invariant open neighborhood $U$ of 0 in $\operatorname{Kur}\left(X_{1}^{e}\right)$, a stack $\left.\left[\left(U \backslash\left(\{0\} \oplus \operatorname{Def}_{2}\right)\right)\right) / \operatorname{Aut}\left(X_{1}^{e}\right)\right]$ has a natural étale morphism to the previously constructed moduli stack so that the glueing is possible. Indeed, the $\mathbb{Q}$-Gorestein deforming component (cf. [39, section 5]) of a Luna étale slice in the Hilbert scheme $\operatorname{Hilb}\left(\mathbb{P}\left(H^{0}\left(X_{1}^{T},-K_{X_{1}^{T}}^{\otimes m}\right)\right)\right)$ at $\left[X_{1}^{T}\right]$ with respect to the standard SL action is étale locally semi-universal deformation by the universality of the Hilbert scheme. Then the étale local uniqueness of semi-universal family tells us it is actually étale locally equivalent with $U$ including the family on it. Then the assertion follows from the universality of Hilbert scheme again. Note that especially $U$ includes the subspace $\operatorname{Def}_{1} \oplus\{0\}$ so that the categorical moduli of the open immersion $\left.\left[\left(U \backslash\left(\{0\} \oplus \operatorname{Def}_{2}\right)\right)\right) / \operatorname{Aut}\left(X_{1}^{e}\right)\right] \hookrightarrow\left[U / \operatorname{Aut}\left(X_{1}^{e}\right)\right]$ represents the contraction of $\mathbb{P}^{1}$.

Then $\mathcal{M}_{1}$ is a KE moduli stack and $M_{1}$ constructed above is KE moduli space. This completes the proof of Theorem 1.1 for degree 1 case as well. Note that our contraction of $\mathbb{P}^{1}$ on the coarse quotient is constructed just on an étale cover, not 
a priori an open substack. Indeed it is not, although we omit the lengthy proof for that. This is the reason our argument is not enough to show $M_{1}$ is a (projective) variety. Completely as before, there is a natural anti-holomophic involution on $M_{1}$ which gives rise to the complex conjugation.

5.3.4. A remark on a conjecture of Corti. In the paper [18, Corti conjectured the following, motivated by the possibility of using birational geometry to get certain "nice" integral models over a discrete valuation ring:

Conjecture 5.29 ([18, Conjecture 1.16]). For an arbitrary smooth punctured curve $C \backslash\{p\}$ and a smooth family of Del Pezzo surfaces $f: \mathcal{X} \rightarrow(C \backslash\{p\})$ over it, we can complete it to a flat family $\bar{f}: \overline{\mathcal{X}} \rightarrow C$ which satisfies:

- $\mathcal{X}$ is terminal.

- $\mathbb{Q}$-Gorenstein index of $\overline{\mathcal{X}}_{p}$ is either $1,2,3$ or 6 and $-6 K_{\overline{\mathcal{X}}_{p}}$ is very ample.

He called $\overline{\mathcal{X}}$ the standard model. We have the following partial solution to the above; it is rather weak, in the sense we permit base change, but on the other hand we even have a classification of the possible central fiber.

Proposition 5.30. For an arbitrary smooth punctured curve $C \backslash\{p\}$ and a smooth family of Del Pezzo surfaces $f: \mathcal{X} \rightarrow(C \backslash\{p\})$ over it, possibly ramified base change $p^{\prime} \in \tilde{C} \rightarrow C$ (with $\left.p^{\prime} \mapsto p\right)$, we can fill the punctured family $\mathcal{X} \times_{(C \backslash\{p\})}\left(\tilde{C} \backslash\left\{p^{\prime}\right\}\right)$ to a flat family $\overline{\mathcal{X}}^{\prime} \rightarrow \tilde{C}$ such that:

- $\overline{\mathcal{X}}^{\prime}$ is terminal.

- $\mathbb{Q}$-Gorenstein index of $\overline{\mathcal{X}}_{p^{\prime}}^{\prime}$ is either 1,2 and $-6 K_{\overline{\mathcal{X}}_{p^{\prime}}^{\prime}}$ is very ample.

Proof. We have constructed the moduli stack $\mathcal{M}_{1}^{\prime \prime}$ by gluing quotient stacks of certain GIT semistable locus (subsection 5.3.2). From the construction, it is universally closed stack and it parametrizes log del Pezzo surfaces of $\mathbb{Q}$-Gorenstein index 1, 2. The $\mathbb{Q}$-Gorenstein property of $\mathcal{X}$ follows from our construction as well.

5.3.5. Relation with moduli of curves. We expect the KE moduli variety $M_{1}$ to be a divisor of one of the geometric compactifactions of moduli of curves with genus 4. Especially we suspect that our moduli $M_{1}$ is the prime divisor of $\bar{M}_{4}(a)$ with $\frac{23}{44}<a<\frac{5}{9}$ in [13]. Note that it is the moduli of Hilbert polystable canonical curves.

\section{FURTHER DISCUSSION}

\subsection{Some remarks.}

6.1.1. Lower bound of the Bergman function. The main technical part in the proof of Proposition 2.2 is a uniform lower bound of the Bergman function. Let $(X, J, \omega, L)$ be a polarized Kähler manifold, then for any $k$ there is an induced metric on $H^{0}\left(X, L^{k}\right)$. The Bergman function is defined by

$$
\rho_{k, X}(x)=\sum\left|s_{\alpha}\right|^{2}(x),
$$

where $\left\{s_{\alpha}\right\}$ is any orthonormal basis of $H^{0}\left(X, L^{k}\right)$. Kodaira embedding theorem says that for fixed $X$, and for sufficiently large $k$ the Bergman function is always positive. It is proved in 23 that for a $n$ dimensional Kähler-Einstein Fano manifold $(X, J, \omega)$, we always have $\rho_{k, X}(x) \geq \epsilon$ for some integer $k$ (and thus every positive multiple of $k$ ) and $\epsilon>0$ depending only on $n$. This was named "partial $C^{0}$ estimate" in [70] and it is also proved there for two dimensional case. It was explained in [23] that one may not take $k$ to be all sufficiently large integers, and in our proof of the main theorem we have seen examples, see Remark 5.14. Indeed we found explicitly all the integers $k$ that we need to take in each degree in order to ensure a 
uniform positivity of Bergman function for all Kähler-Einstein Del Pezzo surfaces. (Compare the strong partial $C^{0}$ estimate in [70], Theorem 2.2):

- $d=4,3: \quad k \geq 1$

- $d=2: k=2 l$, with $l \geq 1$;

- $d=1: k=6 l$, with $l \geq 1$.

6.1.2. Kähler-Einstein metrics on del Pezzo orbifolds. As a consequence of our main Theorem 1.1, we have a complete classification of Kähler-Einstein Del Pezzo surfaces with at worst canonical singularities in terms of K-polystability.

Corollary 6.1. Let $X$ be a Del Pezzo surface with at worst canonical singularities. Then

$$
X \text { admits a Kähler-Einstein metric } \Longleftrightarrow X \text { is K-polystable. }
$$

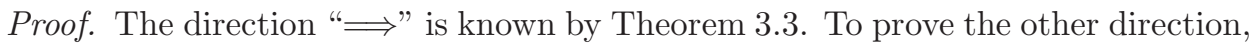
suppose that $X$ is K-polystable and with at worst canonical singularities (in particular it is automatically $\mathbb{Q}$-Gorenstein smoothable). Then by Theorem $3.4 X$ is also polystable with respect to the stability notions that we used in the construction of our moduli spaces, i.e. $[X] \in M_{d}$. Thus $X$ admits a Kähler-Einstein metric as a consequence of Theorem 1.1 .

The above result gives the answer to the conjecture of Cheltsov and Kosta ([16, Conjecture 1.19) on the existence of Kähler-Einstein metrics on canonical Del Pezzo surfaces. In particular, we have the following exact list of possible singularities that can occur. Let $(X, \omega)$ be a degree $d \leq 4$ Del Pezzo surface with canonical singularities, then it admits a Kähler-Einstein metric if and only if $X$ is smooth or

- $d=4: \operatorname{Sing}(X)$ consists of only two $A_{1}$ singularities and $X$ is simultaneously diagonalizable, or exactly four singularities (in which case $X$ is isomorphic to $\left.X_{4}^{T}\right)$

- $d=3: \operatorname{Sing}(X)$ consists of only points of type $A_{1}$, or of exactly three points of type $A_{2}$ (in which case $X$ is isomorphic to $X_{3}^{T}$ );

- $d=2$ : $\operatorname{Sing}(X)$ consists of only points of type $A_{1}, A_{2}$, or of exactly two $A_{3}$ singularities;

- $d=1: \operatorname{Sing}(X)$ consists of only points of type $A_{k}(k \leq 7)$, or of exactly two $D_{4}$ singularities, and $X$ is not isomorphic to one the surfaces in the $\mathbb{P}^{1}$ family in the last section.

As we have seen, the class of log Del Pezzo surfaces with canonical singularities is not sufficient to construct a KE moduli variety. In particular we have found some $\mathbb{Q}$ Gorenstein smoothable Kähler-Einstein log Del Pezzo surfaces, hence K-polystable, with non-canonical singularities. Thus it is natural to ask the following differential geometric/algebro-geometric question: do there exist other $\mathbb{Q}$-Gorenstein smoothable Kähler-Einstein/K-polystable log Del Pezzo surfaces besides the ones which appear in our KE moduli varieties? If the answer to the above question is negative (as we conjecture) then the Yau-Tian-Donaldson conjecture for K-polystability also holds for the class of $\mathbb{Q}$-Gorenstein smoothable Del Pezzo surfaces. For this it is of course sufficient to prove the following: let $\pi: \mathcal{X} \rightarrow \Delta$ be a $\mathbb{Q}$-Gorenstein deformation of a K-polystable Del Pezzo surface $X_{0}$ over the disc $\Delta$ such that the generic fibers $X_{t}$ are smooth (hence admit Kähler-Einstein metrics). Then $X_{0}$ admits a Kähler-Einstein metric $\omega_{0}$, and $\left(X_{0}, \omega_{0}\right)$ is the Gromov-Hausdorff limit of a sequence of Kähler-Einstein metrics on the fibers $\left(X_{t_{i}}, \omega_{t_{i}}\right)$ for some sequence $t_{i} \rightarrow 0$. 
6.2. On compact moduli spaces. In this final section, we would like to formulate a conjecture about the existence of certain compact moduli spaces of Kpolystable/Kähler-Einstein Fano varieties. Before stating our conjecture, we recall some important steps in the history of the construction and compactifications of moduli spaces of varieties.

For complex curves of genus $g \geq 2$, the construction of the moduli spaces, and their "natural" compactifications, was completed during the seventies by Deligne, Mumford, Gieseker and others using GIT. The degenerate curves appearing in the compactification are the so-called "stable curves", i.e., curves with nodal singularities and discrete automorphisms group. Let us recall that these compact moduli spaces have also a "differential geometric" interpretation. It is classically wellknown that every curve of genus $g$ has a unique metric of constant Gauss curvature with fixed volume. As the curves move towards the boundary of the DeligneMumford compactification, the diameters, with respect to the constant curvature metrics, go to infinity and finally these metric spaces "converge" to a complete metric with constant curvature and hyperbolic cusps on the smooth part of a "stable curve".

The construction of compact moduli spaces of higher dimensional polarized varieties turns out to be much more complicated than in the one dimensional cases. Indeed, in the seminal paper 39] the authors discovered examples of surfaces with ample canonical class and semi-log-canonical singularities, which are the natural singularities to be considered for the compactification, which are not asymptotically GIT stable. The central point for this phenomenon is that there are semilog-canonical singularities which have "too big" multiplicity compared to the one required to be asymptotically Chow stable ([54). Nevertheless, proper separated moduli of canonical models of surface of general type have been recently constructed using birational geometric techniques instead of classical GIT. These compactifications are sometimes known as Kollár-Shepherd-Barron-Alexeev (KSBA) type moduli. It is then natural to ask what is the "differential geometric" interpretation of these kind of moduli spaces.

In order to discuss this last point, we first recall that GIT theory became again a main theme for the following reason: the existence of a Kähler-Einstein, or more generally constant scalar curvature, metric on a polarized algebraic variety is found to be deeply linked to some GIT stability notions, e.g., asymptotic Chow, Hilbert stability and in particular to the formally GIT-like notion of "K-stability" introduced in [71, 22]. Similarly to the previous discussion, asymptotic Chow stability seems to not fully capture the existence of a Kähler-Einstein metric since there are examples of Kähler-Einstein varieties which are asymptotically Chow unstable ([39], [58]). On the other hand, for $\mathbb{Q}$-Fano varieties it is indeed proved that the existence of a Kähler-Einstein metric implies K-polystability [11.

It turns out that the notion of K-stability is also closely related with the singularities allowed in the KSBA compactifications ([57, [58]): for varieties with ample canonical class, the notion of K-stability coincides with the semi-log-canonicity property, and for Fano varieties K-(semi)stability implies log-terminalicity. This last condition on the singularities in the Fano case it is also important for differential geometric reasons. As recently shown in [23, it is known that GromovHausdorff limits of smooth Kähler-Einstein Fano manifolds (and more generally of polarized Kähler manifolds with control on the Ricci tensor, the injectivity radius and with bounded diameter) are indeed $\mathbb{Q}$-Fano varieties, i.e., they have at worst log-terminal singularities, and moreover they must be K-polystable, by [11].

Summing up, a central motivation of the present work was to investigate how Kähler-Einstein metrics and the compact moduli varieties are indeed related. Thus, 
motivated by our results on Del Pezzo surfaces and by the above discussion, we shall now try to state a conjecture on moduli of Kähler-Einstein/K-polystable Fano varieties.

Denote the category of algebraic schemes over $\mathbb{C}$ by $\mathrm{Sch}_{\mathbb{C}}$, and let

$$
\mathcal{F}_{h}: \operatorname{Sch}_{\mathbb{C}}^{o} \rightarrow \text { Set }
$$

be the contravariant moduli functor which sends an object $S \in O b\left(\operatorname{Sch}_{\mathbb{C}}\right)$ to isomorphic classes of $\mathbb{Q}$-Gorenstein flat families $\mathcal{X} \rightarrow S$ of K-semistable $\mathbb{Q}$-Fano varieties with Hilbert polynomial equal to $h$ and sends a morphism to pull-back of families making the corresponding squared diagram commuting. Moreover, adding isomorphism (or isotropy) structure on this functor, we should naturally get a stack $\mathcal{M}_{h}$ on which we conjecture, refining [66, Conjecture 1.3.1] and [56, Conjecture 5.2] in the $\mathbb{Q}$-Fano case, the following:

Conjecture 6.2. $\mathcal{M}_{h}$ is a KE moduli stack (cf. Definition 3.13) which has a categorical moduli algebraic space

$$
\mathcal{M}_{h} \rightarrow M_{h},
$$

where $M_{h}$ is a projective variety (in general may not be irreducible) endowed with an ample CM line bundle. Especially, $M_{h}$ is a KE moduli variety in the sense of Definition 3.13

Let $M_{h}^{G H}$ be the Gromov-Hausdorff compactification of the moduli space of smooth Kähler-Einstein Fano manifolds with Hilbert polynomial h. Then there is a natural homeomorphism

$$
\Phi: M_{h}^{G H} \rightarrow M_{h}
$$

where we use the analytic topology on $M_{h}$.

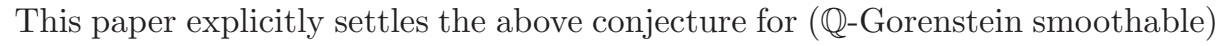
log del Pezzo surface case, except the issue in the previous subsection and the statement about the $\mathrm{CM}$ line bundle. A remark is that the CM line bundle [60] can be naturally regarded as a line bundle on $\mathcal{M}_{h}$ and so by "CM line bundle on $M_{h}$ " we mean a $\mathbb{Q}$-line bundle descended from $\mathcal{M}_{h}$. The descent is possible for each $U_{i} \rightarrow U_{i} / / G$ in the context of Definition 3.13 since for each K-semistable $x \in U_{i}$ the action of the identity component of the isotropy group of $G$ on the CM line over $x$ is trivial by the weight interpretation of vanishing of Futaki invariant [60]. They canonically patch together due to the canonical uniqueness of the descended line bundle on each $U_{i} / / G$.

From the point of view of the authors, one way towards establishing the above conjecture in higher dimensions is by combining the algebraic and differential geometric techniques, as we did in this article. In many concrete situations one can hope to construct the above KE moduli stack by glueing together quotient stacks from different GIT. This also fits into the general conjecture on Artin stack 1, Conjecture 1].

Finally we remark that the points in the boundary $M_{h} \backslash M_{h}^{0}$ should correspond to $\mathbb{Q}$-Fano varieties, admitting weak Kähler-Einstein metrics in the sense of pluripotential theory 24]. This is known for $M_{h}^{G H} \backslash M_{h}^{0}$, see [23].

\section{REFERENCES}

[1] J. Alper. On the local structure of Artin stacks. Journal of Pure and Applied Algebra, Vol. 214, Issue 9, (2010) pp. 1576-1591.

[2] J. Alper, A. Kresch, Equivariant versal deformations of semistable curves, preprint available on his webpage.

[3] V. Alexeev, V. Nikulin. Del Pezzo and K3 surfaces. MSJ Memoirs, 15. Mathematical Society of Japan, Tokyo, 2006. 
[4] M. Anderson. Ricci curvature bounds and Einstein metrics on compact manifolds. J. Amer. Math. Soc. 2 (1989), no. 3, 455-490.

[5] M. Artin. Algebraization of Formal Moduli I, Global Analysis (Papers in Honor of K. Kodaira) pp. 21-71 Univ. Tokyo Press, Tokyo.

[6] M. Artebani, A compactification of $\mathcal{M}_{3}$ via K3 surfaces, Nagoya Math. J. vol. 196 (2009), $1-26$.

[7] S. Bando, A. Kasue, H. Nakajima. On a construction of coordinates at infinity on manifolds with fast curvature decay and maximal volume growth. Invent. Math. 97 (1989), no. 2, 313-349.

[8] S. Bando, T. Mabuchi. Uniqueness of Einstein Kähler metrics modulo connected group actions. Algebraic geometry, Sendai, 1985, 11-40.

[9] K. Behrend, B. Conrad, D. Edidin, W. Fulton, B. Fantechi, L. Göttsche, Andrew Kresch, Algebraic Stacks, In Andrew Kresch's homepage.

[10] D. Burago, Y. Burago, and S. Ivanov, A Course in Metric Geometry. Graduate Studies in Mathematics, vol.33. A.M.S., Providence, RI, (2001).

[11] R. Berman. K-polystability of $\mathbb{Q}$-Fano varieties admitting Kähler-Einstein metrics, arXiv: 1205.6214

[12] J. Borzellino. Orbifolds of maximal diameter. Indiana Univ. Math. J. 42 (1993), no. 1, 37-53.

[13] S. Casalaina-Martin, D. Jensen, R. Laza. Log canonical models and variation of GIT for genus four canonical curves, arXiv:1203.5014.

[14] S. Casalaina-Martin, R. Laza. Simultaneous semistable reduction for curves with ADE singularities. Trans. Amer. Math. Soc. 365 (2013), no. 5, 2271-2295.

[15] I. Cheltsov. On singular cubic surfaces. Asian J. Math. 13 (2009), no. 2, 191-214.

[16] I. Cheltsov, Kosta. Computing $\alpha$-invariants of singular Del Pezzo surfaces. J. Geom. Anal. 24 (2014), no. 2, 798-842.

[17] X-X. Chen, B. Wang. Kähler Ricci flow on Fano manifolds(I). J. Eur. Math. Soc. 14 (2012), no. 6, 2001-2038.

[18] A. Corti, Del Pezzo surfaces over Dedekind schemes, Ann. of Math. 144 (1996), 641-683.

[19] J-P. Demailly. Multiplier ideal sheaves and analytic methods in algebraic geometry, School on Vanishing Theorems and Effective Results in Algebraic Geometry (Trieste, 2000), 1-148.

[20] W-Y. Ding, G. Tian. Kähler-Einstein metrics and the generalized Futaki invariant, Invent. Math., 110, 315-335 (1992).

[21] I. Dolgachev. Lectures on invariant theory. London Mathematical Society Lecture Note Series, 296. Cambridge University Press, Cambridge, 2003.

[22] S. K. Donaldson. Scalar curvature and stability of toric varieties. J. Differential Geom. 62 (2002), no. 2, 289-349.

[23] S. K. Donaldson, S. Sun. Gromov-Hausdorff limits of Kähler manifolds and algebraic geometry, Acta Math. 213 (2014), no.1, 63-106.

[24] P. Eyssidieux, V. Guedj, A. Zeriahi. Singular Kähler-Einstein metrics. J. Amer. Math. Soc. 22 (2009), no. 3, 607-639.

[25] B. Fantechi et. al. Fundamental Algebraic Geometry -Grothendieck's FGA explained-, Mathematical Surveys and Monographs, vol. 123, American Mathematical Society.

[26] M. Furushima. Singular del Pezzo surfaces and analytic compactifications of 3-dimensional complex affine space $\mathbb{C}^{3}$. Nagoya Math. J. 104 (1986), 1-28.

[27] A. Ghigi, J. Kollár. Kähler-Einstein metrics on orbifolds and Einstein metrics on spheres Commentarii Mathematici Helvetici, 82 (2007), 877-902

[28] H. Grauert. Der Satz von Kuranishi für kompakte komplexe Räume. Invent. Math. 25 (1974), $107-142$.

[29] P. Hacking, Y. Prokhorov. Degenerations of Del Pezzo surfaces I, arXiv:math/0509529.

[30] P. Hacking, Y. Prokhorov. Smoothable Del Pezzo surfaces with quotient singularities. Compos. Math. 146 (2010), no. 1, 169-192.

[31] F. Hidaka, K. Watanabe. Normal Gorenstein surfaces with ample anti-canonical divisor. Tokyo J. Math. 4 (1981), no. 2, 319-330.

[32] D. Hilbert. Über die vollen Invariantensysteme. (German) Math. Ann. 42 (1893), no. 3, 313-373.

[33] D. Hyeon, Y. Lee. Log minimal model program for the moduli of stable curves of genus three. Math. Res. Lett. 17 (2010), no. 4, 625-636.

[34] M. Hochster, J.L. Robert, Rings of invariants of reductive groups acting on regular rings are Cohen-Macaulay, Adv. in Math. (1974).

[35] S. Ishii, The canonical modifications by weighted blow ups. J. Algebraic Geom. 5 (1996), no. 4, 783-799.

[36] Y. Kawamata, Crepant blowing-up of 3-dimensional canonical singularities and its application to degenerations of surfaces. Ann. of Math. vol. 127, 93-163 (1988). 
[37] G. Kapustka, M. Kapustka. Equations of log Del Pezzo surfaces of index $\leq 2$. Math. Z. 261 (2009), no. 1, 169-188.

[38] A.M.Kasprzyk, M.Kreuzer, B.Nill, On the combinatorial classification of toric log Del Pezzo surfaces. LMS Journal of Computation and Mathematics 13, 2010, 33-46.

[39] J. Kollár, N. I. Shepherd-Barron. Threefolds and deformations of surface singularities. Invent. Math. 91 (1988), no. 2, 299-338.

[40] P. Kronheimer. The construction of ALE spaces as hyper-Kähler quotients J. Differential Geom. (1989), no. 3, 665-683.

[41] D. Luna Slices étales, Sur les groupes algébriques, Bull. Soc. Math. France, Paris, Mémoire 33 (1973).

[42] C. LeBrun, S.R. Simanca. Extremal Kähler metrics and complex deformation theory. Geom. Funct. Anal. 4 (1994), no. 3, 298-336.

[43] C. Li, C-Y. Xu. Special test configuarions and K-stability of $\mathbb{Q}$-Fano varieties. Ann. of Math. (2) 180 (2014), no. 1, 197-232.

[44] T. Mabuchi. K-stability of constant scalar curvature polarization, arxiv: 0812.4093.

[45] T. Mabuchi, S. Mukai. Stability and Einstein-Kähler metric of a quartic Del Pezzo surface. Einstein metrics and Yang-Mills connections (Sanda, 1990), 133-160, Lecture Notes in Pure and Appl. Math., 145, Dekker, New York, 1993.

[46] M. Manetti. Normal degenerations of the complex plane. J. Reine Angew. Math. 419(1991), 89-118.

[47] M. Manetti. $\mathbb{Q}$-Gorenstein smoothings of quotient singularities. Preprint Scuola Normale Superiore Pisa (1990).

[48] M. Manetti. Degenerations of Algebraic Surfaces and applications to Moduli problems. PhD thesis, Scuola Normale Superiore, Pisa (1995).

[49] H. Matsumura, Commutative Algebra, Benjamin, second edition (1980)

[50] Y. Matsushima. Sur la structure du groupe d'homéomorphismes analytiques d'une certaine variété kählérienne. Nagoya Math. J. 11 (1957), 145-150

[51] R. Miranda. The Moduli of Weierstrass Fibrations over $\mathbb{P}^{1}$, Math. Ann. 255 (1981), 379-394.

[52] S. Mukai. New developments in Fano manifold theory related to the vector bundle method and moduli problems. (Japanese) Sugaku 47 (1995), no. 2, 125-144.

[53] D.Mumford, J. Fogarty, F. Kirwan, Geometric Invariant Theory, Ergebnisse Der Mathematik Und Ihrer Grenzgebiete. 2. Folge.

[54] D. Mumford, Stability of projective varieties, Enseignement Math. 23 (1977)

[55] N. Nakayama. Classification of log Del Pezzo surfaces of index two. J. Math. Sci. Univ. Tokyo 14 (2007), no. 3, 293-498.

[56] Y.Odaka. On the GIT stability of Polarized Varieties - a survey-, Proceeding of Kinosaki algebraic geometry symposium 2010. available online.

[57] Y.Odaka. The GIT stability of Polarized Varieties via Discrepancy. . Ann. of Math. (2) 177 (2013), no. 2, 645-661.

[58] Y.Odaka. The Calabi conjecture and K-stability, Int. Math. Res. Notices. issue 13. (2011).

[59] Y.Odaka. On parametrization, optimization and triviality of test configurations, arXiv:1201.0692.

[60] S. Paul, G. Tian. CM stability and the generalized Futaki invariant II Astérisque No. 328 (2009), 339-354 (2010).

[61] D. H. Phong, J. Ross, and J. Sturm. Deligne pairings and the Knudsen-Mumford expansion J. Differential Geom. Volume 78, Number 3 (2008), 475-496.

[62] D. Rim. Equivariant G-structure on versal deformations. Trans. Amer. Math. Soc. 257 (1980), $217-226$.

[63] G. Salmon. A treatise on the analytic geometry of three dimensions. Vol. II. Fifth edition. Edited by Reginald A. P. Rogers Chelsea Publishing Co., New York 1965

[64] J. Shah. A complete moduli space for K3 surfaces of degree 2. Ann. of Math. (2) 112 (1980), no. 3, 485-510.

[65] Y-L. Shi. On the $\alpha$-invariants of cubic surfaces with Eckardt points. Adv. Math. 225 (2010), no. 3, 1285-1307

[66] C. Spotti. Ph. D. Thesis, Imperial College London, 2012.

[67] C. Spotti. Deformations of Nodal Kähler-Einstein Del Pezzo Surfaces with Finite Automorphism Groups J. London Math. Soc. (2014) 89 (2).

[68] J. Stoppa. K-stability of constant scalar curvature Kähler manifolds. Adv. Math. 221 (2009), no. 4, 1397-1408

[69] I. Suvaina. ALE Ricci-flat Kähler metrics and deformations of quotient surface singularities Ann. Global Anal. Geom. (2012), no. , 109-123.

[70] G. Tian. On Calabi's conjecture for complex surfaces with positive first Chern class. Invent. Math. 101 (1990), no. 1, 101-172. 
[71] G. Tian. Kähler-Einstein metrics on algebraic manifolds. Proc. of ICM. Kyoto, 1990.

[72] G. Tian. The K-energy on hypersurfaces and stability Comm. Anal. Geom., vol. 2, 239-265, (1994) iss 2, 239-265.

[73] G. Tian. Kähler-Einstein metrics with positive scalar curvature. Invent. math. 137, 1-37 (1997)

[74] G. Tian. Existence of Einstein metrics on Fano manifolds. Progress in Mathematics, 2012, Volume 297, Part 1, 119-159.

[75] G. Tian, S-T. Yau. Kähler-Einstein metrics on complex surfaces with $c_{1}>0$. Comm. Math. Phys. 112 (1987), no. 1, 175-203.

[76] N. Tziolas. Smoothings of scheme with non-isolated singularities. Michigan Math. J. 59 (2010), no. 1, 25-84.

[77] B. Wang. Ricci flow on orbifolds. arXiv:1003.0151.

[78] X-W. Wang, Heights and GIT weights. Math. Res. Lett. 19 (2012), no. 4, 909-926.

Department of Mathematics, Kyoto University, Kyoto 606-8502, Japan

E-mail address: yodaka@math.kyoto-u.ac.jp

DPmms, Centre for Mathematical Sciences, Wilberforce Road, Cambridge CB3 OWB, United Kingdom

E-mail address: c.spotti@dpmms.cam.ac.uk / cristiano.spotti@gmail.com

Department of Mathematics, Stony Brook University, Stony Brook, Ny 11794-3651, USA

E-mail address: song.sun@stonybrook.edu 